hep-th/0102080

McGill/01-01

\title{
Non-abelian Brane Intersections
}

\author{
Neil R. Constable巴, Robert C. Myers] and Øyvind Tafjord] \\ Department of Physics, McGill University \\ Montréal, QC, H3A 2T8, Canada
}

\begin{abstract}
We study new solutions of the low-energy equations of motion for the non-abelian D-string. We find a "fuzzy funnel" solution consisting of a noncommutative four-sphere geometry which expands along the length of the D-string. We show that this funnel solution has an interpretation as D-strings ending on a set of orthogonal D5-branes. Although not supersymmetric, the system appears to be stable within this framework. We also give a dual description of this configuration as a bion spike in the non-abelian world volume theory of coincident D5-branes.
\end{abstract}

\footnotetext{
${ }^{1}$ E-mail: constabl@hep.physics.mcgill.ca

${ }^{2}$ E-mail: rcm@hep.physics.mcgill.ca

${ }^{3}$ E-mail: tafjord@physics.mcgill.ca
} 


\section{Introduction}

The advent of D-branes [1] has brought about significant advances in string theory — for reviews see refs. [2, 3]. Much of the progress has come about by directly studying the low energy dynamics of the D-brane's world volume which is known to be governed by the BornInfeld action [4, 5]. This world volume theory living on D-branes has many fascinating features. Among these is the possibility for D $p$-branes, through an appropriate excitation of fields, to morph into objects resembling $\mathrm{D} p^{\prime}$-branes of lower or higher dimensionalities. This is seen, for instance, in the Born-Infeld string [6] where a D3-brane, pulled out into a spike and adorned with a magnetic monopole field, describes a D-string extending out from the D3-brane. We also know from matrix theory [0, 8] and the dielectric effect for D-branes [9] that a collection of D0-branes can expand into a D2-brane. This is a direct consequence of the fact that the world volume theory living on a collection of coincident D-branes possesses a non-abelian gauge symmetry [10] and that the world volume fields describing the brane's position become matrix valued in this context. The non-abelian generalization of the Born-Infeld action has received much attention recently. The form of the bosonic part of the action for arbitrary supergravity backgrounds was proposed in refs. 9, 11] and has led to many interesting developments including the giant gravitons of ref. [12], the resolution of curvature singularities a la Polchinski and Strassler [13] as well as several new insights into world volume gauge theories as described, for example, in refs. 114, 15, 16, 17, 18, 19, 20].

In this paper, we will use the non-abelian Born-Infeld action to study a set of D1-branes ending on a collection of orthogonal D5-branes. We find that this D $\perp \perp$ D1 intersection shares many features with the well-known D3 $\perp$ D1 system, but there will, of course, be interesting differences as well. Since the D $5 \perp \mathrm{D} 1$-system is not a brane intersection that is commonly discussed, let us begin by reminding the reader how it is possible for D-strings to end on D5-branes. The D-strings are electric charge sources for the Ramond-Ramond (RR) twoform potential $C^{(2)}$, and so one must understand how this charge is conserved in the D $5 \perp \mathrm{D} 1$ intersection [21]. In the presence of D5-branes, the equations of motion for the RR two-form are modified by the appearance of new source terms due to the following term in the D5-brane action,

$$
S_{W Z} \simeq 2 \pi^{2} \alpha^{\prime 2} \mu_{5} \int_{\mathrm{D} 5} \mathrm{~S} \operatorname{Tr}\left[C^{(2)} \wedge F \wedge F\right] .
$$

Hence D-strings can end on D5-branes, but they must act as sources of second Chern class or instanton number in the world volume theory of the D5-branes [22]. That is, the intersection will be described by a configuration involving the D5-brane gauge field $F$ with $\int F \wedge F \neq 0$, for any four-surface surrounding the D-string endpoints. Hence, one interesting point that distinguishes this from the $\mathrm{D} 3 \perp \mathrm{D} 1$ system, is that if the $\mathrm{D} 5 \perp \mathrm{D} 1$ intersection is to be resolved by a smooth low energy field configuration, it must involve a non-abelian gauge field. This, in turn, indicates that the intersection must involve several coincident D5-branes rather than just a single D5-brane. Another feature, which distinguishes the D5 $\perp \mathrm{D} 1$ intersection from the $\mathrm{D} 3 \perp \mathrm{D} 1$ system, is that it is not supersymmetric. The latter is easily seen in the context of perturbative string theory. The D5-D1 strings would have six Neumann-Dirichlet directions, 
which is inconsistent with a supersymmetric ground-state [2, 3].

In analogy with the previous work on the D3 $\perp$ D1 case [6, 23, 14, we will examine the $\mathrm{D} 5 \perp \mathrm{D} 1$ configuration from two dual points of view. Beginning with the world volume theory of the D-strings, in analogy to the D3 $\perp$ D1 construction in ref. [14], we find a funnel configuration in which the D-strings expand through a noncommutative geometry into a set of D5-branes. The second point of view is constructing the D $\perp \perp \mathrm{D} 1$ intersection within the D5-brane world volume theory. Here we construct a non-abelian gauge field as described above, and we also excite one of the transverse scalars. The D-strings then appear as a bion spike which smoothly deforms the (otherwise) planar geometry of the D5-branes.

The noncommutative space which we encounter in the D-string funnel is the fuzzy four sphere, which we describe using an explicit matrix representation found in ref. [24. Due to a curious restriction on the dimensions of these matrices we find that the number of D-strings, $N$, and the number of D5-branes, $n$, are related by $N \sim n^{3} / 6$ for large $n$. We find a similar relation in the D5-brane world volume description. There our construction of the D5 $\perp$ D1 intersection involves $S U(n)$ instantons which are homogeneous on the four sphere. It turns out that the spherical symmetry imposes an upper bound on the instanton number, $N$, given again by $N \sim n^{3} / 6$ for large $n$ !

We find also that quantities such as the energy and Ramond-Ramond charges of the D $5 \perp \mathrm{D} 1$ intersection agree between the dual D-string and D5-brane points of view at large $N$. While the agreement begins to fail for small $N$ this should not be viewed as a failure of the duality between these two descriptions. Rather it is a limitation of our analysis which relies on the non-abelian Born-Infeld action. The latter is only a low energy action which does not give a reliable description of all field configurations. Hence, for small $N$, our dual analyses give complimentary descriptions of the same system which are valid in different regimes. Of course, similar limitations are also found in examining the D3 $\perp$ D1 intersection [14].

An outline of the paper is as follows: Since many of our considerations are in analogy with previous studies of the D3 $\perp$ D1 system, we give a brief review of the latter in Section 2 . In Section 3, we consider the D $\perp$ D1 system from the point of view of the low energy Dstring theory, while in Section 4, we do the same using the D5-brane world volume theory. In Section 5, we present the extension of our analysis to the case where the D-strings are replaced by dyonic strings, i.e., bound states of fundamental and Dirichlet strings. In Section 6, we consider fluctuations of the static D5 $\perp$ D1 configuration from the point of view of the D-string theory. Finally in Section 7, we provide a discussion of our results. Appendix A summarizes the properties of the matrices used to construct the noncommutative four-sphere. In Appendix $\mathrm{B}$, we give a discussion of the homogeneous instantons which play a role in the D5-brane construction of Section 4. 


\section{Review of the D3 $\perp$ D1-brane system}

In this section, we briefly review a system consisting of a set of D-strings ending on an orthogonal D3-brane. This has two dual descriptions coming from the D3-brane and the D-string world volume theories. Within either of these theories, the field configurations can be determined by simply solving the BPS conditions or by studying the full Born-Infeld equations of motion. Here, we use a simple approach based on minimizing the energy [25], while pointing out some aspects that will be of relevance in later sections.

We start with the D3-brane theory construction. The low energy dynamics of a (single) D3-brane in a Minkowski space background is described by the Born-Infeld action [4],

$$
S=\int d t \mathcal{L}=-T_{3} \int d^{4} \sigma \sqrt{-\operatorname{det}\left(\eta_{a b}+\lambda^{2} \partial_{a} \phi^{i} \partial_{b} \phi^{i}+\lambda F_{a b}\right)},
$$

where $\lambda \equiv 2 \pi \alpha^{\prime}=2 \pi \ell_{s}^{2}$. Here we have implicitly used static gauge, with $\sigma^{a}(a=0, \ldots, 3)$ denoting the world volume coordinates while $\phi^{i}(i=4, \ldots, 9)$ are the scalars describing transverse fluctuations of the brane. Also, $F_{a b}$ is the field strength of the $U(1)$ gauge field on the brane.

Within this theory, D-strings appear as BPS magnetic monopoles [6]. Hence we consider exciting one of the scalar fields, say $\phi \equiv \phi^{9}$, as well as a magnetic field $B^{r}=\frac{1}{2} \epsilon^{r s t} F_{s t}(r, s, t=$ $1, \ldots, 3)$. One can show that it is consistent with the equations of motion to set all the other fields equal to zero. For static configurations, we then evaluate the energy,

$$
\begin{aligned}
E & =-\mathcal{L}=T_{3} \int d^{3} \sigma \sqrt{1+\lambda^{2}|\vec{\nabla} \phi|^{2}+\lambda^{2}|\vec{B}|^{2}+\lambda^{4}(\vec{B} \cdot \vec{\nabla} \phi)^{2}} \\
& =T_{3} \int d^{3} \sigma \sqrt{\lambda^{2}|\vec{\nabla} \phi \mp \vec{B}|^{2}+\left(1 \pm \lambda^{2} \vec{B} \cdot \vec{\nabla} \phi\right)^{2}} \\
& \geq T_{3} \int d^{3} \sigma\left(1 \pm \lambda^{2} \vec{B} \cdot \vec{\nabla} \phi\right) .
\end{aligned}
$$

The first term in this lower bound is simply the energy of the D3-brane. The second term is a total derivative: $\vec{B} \cdot \vec{\nabla} \phi=\vec{\nabla} \cdot(\vec{B} \phi)$ using the Bianchi identity $\vec{\nabla} \cdot \vec{B}=0$. Hence this term is topological, depending only on the boundary values of the fields specified at infinity and near singular points (after introducing a cutoff, such as at $r=0$ below). Therefore, the last line of eq. (3) provides a true minimum of the energy for a given set of boundary conditions.

The lower bound in eq. (3) is achieved when

$$
\vec{\nabla} \phi= \pm \vec{B}
$$

which coincides with the BPS condition for the magnetic monopoles [6]. Further, using the Bianchi identity, eq. (4) implies $\vec{\nabla}^{2} \phi=0$ and so we are looking for harmonic functions on the D3-brane. The simplest solution, corresponding to the "bion spike," is given by

$$
\phi(r)=\frac{N}{2 r}, \quad \vec{B}(\vec{r})=\mp \frac{N}{2 r^{3}} \vec{r},
$$


where $r^{2}=\left(\sigma^{1}\right)^{2}+\left(\sigma^{2}\right)^{2}+\left(\sigma^{3}\right)^{2}$, and $N$ is an integer due to quantization of the magnetic charge. The energy of this configuration is readily computed to be

$$
E=T_{3} \int d^{3} \sigma+N T_{1} \int_{0}^{\infty} d(\lambda \phi)
$$

where $T_{1}=\left(2 \pi \ell_{s}\right)^{2} T_{3}$. Hence we have recovered the energy expected for a BPS configuration consisting of $N$ semi-infinite D-strings ending on an orthogonal D3-brane. Note that the physical distance in the transverse direction is $\lambda \phi$. Other considerations, such as the effective charge distribution and fluctuations on the strings [26], confirm that the bion spike describes the D3 $\perp$ D1-system to a good approximation.

Now we consider the dual description of the D3 $\perp$ D1-system given by the D-string theory. The low energy dynamics of $N$ D-strings in a flat background is well described by the nonabelian Born-Infeld action [9, 5]:

$$
S=-T_{1} \int d^{2} \sigma \operatorname{STr} \sqrt{-\operatorname{det}\left(\eta_{a b}+\lambda^{2} \partial_{a} \Phi^{i} Q_{i j}^{-1} \partial_{b} \Phi^{j}\right) \operatorname{det}\left(Q^{i j}\right)},
$$

where $Q^{i j}=\delta^{i j}+i \lambda\left[\Phi^{i}, \Phi^{j}\right]$. Again we are assuming static gauge where the two worldsheet coordinates are identified with $\tau=x^{0}$ and $\sigma=x^{9}$. The transverse scalars $\Phi^{i}(i=1, \ldots, 8)$ are now $N \times N$ matrices transforming in the adjoint representation of the $U(N)$ worldsheet gauge symmetry. The symmetrized trace prescription [27], denoted by STr, requires that we symmetrize over all permutations of $\partial_{a} \Phi^{i}$ and $\left[\Phi^{i}, \Phi^{j}\right]$ within the gauge trace upon expanding the square root. For explicit computations, it is often convenient to write the two determinants under the square root in terms of the single determinant,

$$
S=-T_{1} \int d^{2} \sigma \operatorname{STr}\left[-\operatorname{det}\left(\begin{array}{cc}
\eta_{a b} & \lambda \partial_{a} \Phi^{j} \\
-\lambda \partial_{b} \Phi^{i} & Q^{i j}
\end{array}\right)\right]^{\frac{1}{2}} .
$$

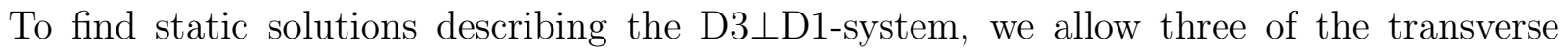
scalars, $\Phi^{i}(i=1,2,3)$, to depend on the spatial coordinate $\sigma$. Evaluating the determinant in eq. (8) the energy becomes

$$
\begin{aligned}
E & =T_{1} \int d \sigma \mathrm{S} \operatorname{Tr} \sqrt{1+\lambda^{2}\left(\partial_{\sigma} \Phi^{i}\right)^{2}-\frac{1}{2} \lambda^{2}\left[\Phi^{i}, \Phi^{j}\right]^{2}-\frac{1}{4} \lambda^{4}\left(\epsilon^{i j k} \partial_{\sigma} \Phi^{i}\left[\Phi^{j}, \Phi^{k}\right]\right)^{2}} \\
& =T_{1} \int d \sigma \mathrm{S} \operatorname{Tr} \sqrt{\lambda^{2}\left(\partial_{\sigma} \Phi^{i} \mp \frac{i}{2} \epsilon^{i j k}\left[\Phi^{j}, \Phi^{k}\right]\right)^{2}+\left(1 \pm \frac{i}{2} \lambda^{2} \epsilon^{i j k} \partial_{\sigma} \Phi^{i}\left[\Phi^{j}, \Phi^{k}\right]\right)^{2}} \\
& \geq T_{1} \int d \sigma \mathrm{S} \operatorname{Tr}\left(1 \pm \frac{i}{2} \lambda^{2} \epsilon^{i j k} \partial_{\sigma} \Phi^{i}\left[\Phi^{j}, \Phi^{k}\right]\right) \\
& =N T_{1} \int d \sigma \pm \frac{i}{3} \lambda^{2} T_{1} \int d \sigma \partial_{\sigma} \operatorname{Tr}\left(\epsilon^{i j k} \Phi^{i} \Phi^{j} \Phi^{k}\right) .
\end{aligned}
$$

Here the lower bound is again the sum of a trivial term (the energy of the $N$ D-strings) and a topological term. The minimum energy condition is

$$
\partial_{\sigma} \Phi^{i}= \pm \frac{i}{2} \epsilon^{i j k}\left[\Phi^{j}, \Phi^{k}\right]
$$


which can be identified as the Nahm equations [28, 23]. The desired solution is given by

$$
\Phi^{i}(\sigma)= \pm \frac{\alpha^{i}}{2 \sigma}
$$

where the $\alpha^{i}$ are an $N \times N$ representation of the $S U(2)$ algebra,

$$
\left[\alpha^{i}, \alpha^{j}\right]=2 i \epsilon^{i j k} \alpha^{k} .
$$

These $S U(2)$ matrices satisfy $\sum\left(\alpha^{i}\right)^{2}=C \mathbf{1}_{N}$, where $\mathbf{1}_{N}$ the $N \times N$ identity matrix, and the constant $C$ is the Casimir. We focus on the irreducible $N \times N$ representation for which $C=N^{2}-1$. This noncommutative scalar field configuration (11) describes a fuzzy two-sphere 29 with a physical radius

$$
R(\sigma)=\lambda \sqrt{\frac{1}{N} \operatorname{Tr}\left[\Phi^{i}(\sigma)^{2}\right]}=\frac{\sqrt{C} \pi \ell_{s}^{2}}{\sigma}=\frac{N \pi \ell_{s}^{2}}{\sigma} \sqrt{1-1 / N^{2}} .
$$

Hence the solution describes a "fuzzy funnel" in which the D-strings expand to fill the $X^{1,2,3}$ hyperplane at $\sigma=0$. This geometry can be compared to the D3-brane solution (5) after relabeling $\sigma \rightarrow \lambda \phi$ and $R \rightarrow r$. We see that both descriptions yield the same geometry in the limit of large $N$, up to $1 / N^{2}$ corrections. There is similar agreement for other quantities, such as the energy,

$$
E=N T_{1} \int_{0}^{\infty} d \sigma+\left(1-1 / N^{2}\right)^{-1 / 2} T_{3} \int 4 \pi R^{2} d R
$$

and the charges, in the large $N$ limit. Hence at least in this limit, the two dual descriptions are in good agreement, as might be expected since the fuzziness of the funnel solution is effectively smoothed out. Note, however, that the Born-Infeld actions, eqs. (2) and (7), only describe low energy dynamics and do not provide a complete theory in either case. Therefore, in general, one must regard these two dual descriptions as complimentary. That is, the D-string theory gives reliable results near the core of the spike, while the D3-brane theory is valid for $r$ large - see ref. [14 for further discussion of these issues.

\section{D-strings growing into D5-branes}

As we reviewed above and elaborated on in ref. [14], the non-abelian theory describing $N$ coincident D-strings has noncommutative scalar field solutions which have an interesting interpretation in terms of fuzzy geometry. To describe the D3 $\perp$ D1-system, it is natural to consider a funnel with a fuzzy two-sphere as the cross-section. However, this particular noncommutative geometry was introduced by hand, and so a natural question to investigate is whether similar solutions of the D-string equations of motion exist which involve fuzzy geometries other than the two-sphere. In the following, we focus on a generalization involving fuzzy four-spheres based on the matrices constructed by Castelino, Lee and Taylor 24. As might be expected from their analysis, we will see that this construction leads to a fuzzy funnel in which the 
D-strings expand into orthogonal D5-branes. Although not supersymmetric, this configuration shares many common features with the D3-brane funnel. We will comment briefly on other noncommutative geometries in the discussion section.

Our starting point is again the low energy action for $N$ D-strings (7), however, we now consider static configurations involving five (rather than three) nontrivial scalars, $\Phi^{i}$ with $i=$ $1, \ldots, 5$. In this case, the action becomes

$$
\begin{aligned}
S= & -T_{1} \int d^{2} \sigma \operatorname{STr}\left\{1+\lambda^{2}\left(\partial_{\sigma} \Phi^{i}\right)^{2}+2 \lambda^{2} \Phi^{i j} \Phi^{j i}+2 \lambda^{4}\left(\Phi^{i j} \Phi^{j i}\right)^{2}-4 \lambda^{4} \Phi^{i j} \Phi^{j k} \Phi^{k l} \Phi^{l i}+\right. \\
& \left.+2 \lambda^{4}\left(\partial_{\sigma} \Phi^{i}\right)^{2} \Phi^{j k} \Phi^{k j}-4 \lambda^{4} \partial_{\sigma} \Phi^{i} \Phi^{i j} \Phi^{j k} \partial_{\sigma} \Phi^{k}+\frac{\lambda^{6}}{4}\left(\epsilon^{i j k l m} \partial_{\sigma} \Phi^{i} \Phi^{j k} \Phi^{l m}\right)^{2}\right\}^{1 / 2},
\end{aligned}
$$

where we have introduced the convenient notation

$$
\Phi^{i j} \equiv \frac{1}{2}\left[\Phi^{i}, \Phi^{j}\right]
$$

To construct a new funnel solution, we consider the following ansatz:

$$
\Phi^{i}(\sigma)= \pm \hat{R}(\sigma) G^{i}, \quad i=1, \ldots, 5
$$

where $\hat{R}(\sigma)$ is the (positive) radial profile and $G^{i}$ are the matrices constructed in ref. 24] - see also ref. [30]. We give the definition and many useful properties of the $G^{i}$ matrices in appendix A. Here, we simply note that the $G^{i}$ are given by the totally symmetric $n$-fold tensor product of $4 \times 4$ gamma matrices, and that the dimension of the matrices is related to the integer $n$ by

$$
N=\frac{(n+1)(n+2)(n+3)}{6} .
$$

The solution will describe a funnel whose cross-section is a fuzzy four-sphere with a physical radius

$$
R(\sigma)=\lambda \sqrt{\operatorname{Tr}\left[\Phi^{i}(\sigma)^{2}\right] / N}=\lambda \hat{R}(\sigma) \sqrt{\operatorname{Tr} G^{i} G^{i} / N}=\sqrt{c} \lambda \hat{R}(\sigma) .
$$

The constant $c$ is the "Casimir" associated with the $G^{i}$ matrices, i.e., $G^{i} G^{i}=c \mathbf{1}_{N}$, given by

$$
c=n(n+4) \text {. }
$$

Before considering the full solution, we remind the reader of a puzzle presented in ref. [14]. Consider the lowest order equations of motion,

$$
\left(-\partial_{\tau}^{2}+\partial_{\sigma}^{2}\right) \Phi^{i}=\left[\Phi^{j},\left[\Phi^{j}, \Phi^{i}\right]\right]
$$

which are independent of the number of transverse scalars being excited. Substituting in the static ansatz (17), and applying various matrix identities presented in the appendix, yields

$$
\hat{R}^{\prime \prime}=16 \hat{R}^{3}
$$


which yields a simple funnel solution: $\hat{R}(\sigma)=(2 \sqrt{2} \sigma)^{-1}$. Now, this solution shows the same $R \sim \sigma^{-1}$ behavior as we found for the D3-brane funnel (11). In fact, since the lowest order equations always take the same form, this will be the universal small $R$ behavior of any D-string funnel. However, if the funnel constructed here is to expand into a D5-brane, we expect that at large $R$, the expansion would be governed by a harmonic function in five spatial dimensions, i.e., $\sigma \sim R^{-3}$ or $R \sim \sigma^{-1 / 3}$. In ref. [14] we anticipated the resolution of this puzzle, namely, that higher order terms in the Born-Infeld action would effect a transition from the universal small- $R$ behavior to the "harmonic" expansion at large $R$. This is precisely the result that we find in the following.

Now consider the full equations of motion resulting from the action (15). It is a straightforward but tedious exercise to calculate the latter, and the result is a rather formidable and unilluminating expression that we do not include here. However, plugging the ansatz (17) into these equations, one finds that all the matrix products simplify, leaving a single overall factor of $G^{i}$. To verify this claim one uses the identities given in appendix A. The final result is then simply a differential equation for $\hat{R}(\sigma)$.

Knowing this, a simpler procedure is to substitute the ansatz (17) directly into the action (15). We again make heavy use of the matrix identities in appendix A, and we choose to express the results in terms of the physical radius $R$, rather than $\hat{R}$, using eq. (19). The resulting action for the radial profile $R(\sigma)$ is

$$
S=-N T_{1} \int d^{2} \sigma \sqrt{1+\left(R^{\prime}\right)^{2}}\left[1+4 R^{4} /\left(c \lambda^{2}\right)\right] .
$$

Actually this result is only captures the leading large- $N$ contribution at each order in the expansion of the square root. There are corrections at order $1 / c$ because we have not fully implemented the symmetrization of the trace.

From the action (23), we can derive an energy bound similar to that in eq. (9),

$$
\begin{aligned}
E & =N T_{1} \int d \sigma\left[\left(R^{\prime} \mp \sqrt{8 R^{4} /\left(c \lambda^{2}\right)+16 R^{8} /\left(c \lambda^{2}\right)^{2}}\right)^{2}+\left(1 \pm R^{\prime} \sqrt{8 R^{4} /\left(c \lambda^{2}\right)+16 R^{8} /\left(c \lambda^{2}\right)^{2}}\right)^{2}\right]^{1 / 2} \\
& \geq N T_{1} \int d \sigma\left(1 \pm R^{\prime} \sqrt{8 R^{4} /\left(c \lambda^{2}\right)+16 R^{8} /\left(c \lambda^{2}\right)^{2}}\right) .
\end{aligned}
$$

This is again a sum of the trivial term and a topological term. The equality is obtained when

$$
R^{\prime}=\mp \sqrt{8 R^{4} /\left(c \lambda^{2}\right)+16 R^{8} /\left(c \lambda^{2}\right)^{2}} .
$$

Note that this equation is also compatible with the full equation of motion — see end of the section for more discussion.

Let us now investigate the profile specified by eq. (25). This equation can be explicitly solved in terms of elliptic functions, but it is more instructive to consider various limits. For small $R$, the $R^{4}$ term under the square root dominates, and we find the funnel solution

$$
R(\sigma) \simeq \frac{\sqrt{c} \lambda}{2 \sqrt{2} \sigma}
$$


This is precisely the leading order solution found above with the universal behavior: $R \sim \sigma^{-1}$. However, for large $R$ the equation becomes $R^{\prime}=\mp 4 R^{4} /\left(c \lambda^{2}\right)$, with solution

$$
R(\sigma) \simeq\left(\frac{c \lambda^{2}}{12 \sigma}\right)^{1 / 3}
$$

which is precisely the harmonic behavior that we anticipated for a D5-brane to appear at $\sigma=0$. The cross-over between the universal and harmonic expansion occurs when the two terms under the square root are comparable, i.e.,

$$
R_{c} \sim\left(c \lambda^{2} / 2\right)^{1 / 4}=\left(2 \pi^{2} c\right)^{1 / 4} \ell_{s}
$$

Note that for large $c$ (and hence large $N$ ), this is a macroscopic distance scale, i.e., $R_{c} \gg \ell_{s}$.

To verify that the solution of eq. (25) indeed corresponds to the funnel expanding into a number of D5-branes, we can investigate the RR charge and the energy. If the configuration corresponds to D5-branes located at $\sigma=0$ and spanning the $X^{1,2,3,4,5}$ directions, it should be a source for the six-form RR potential $C_{012345}^{(6)}$. The required source term comes from the following term in the non-abelian Wess-Zumino action [9, 11,

$$
\begin{aligned}
-\frac{\lambda^{2} \mu_{1}}{2} \int \mathrm{S} \operatorname{Tr} P\left[\left(\mathrm{i}_{\Phi} \mathrm{i}_{\Phi}\right)^{2} C^{(6)}\right] & =-\frac{\lambda^{3} \mu_{1}}{2} \int d \sigma d \tau C_{012345}^{(6)} \operatorname{STr}\left(\epsilon^{i j k l m} \Phi^{i} \Phi^{j} \Phi^{k} \Phi^{l} \partial_{\sigma} \Phi^{m}\right) \\
& = \pm \frac{6 N(n+2)}{c^{3 / 2}} \mu_{5} \int d \tau d R \Omega_{4} R^{4} C_{012345}^{(6)},
\end{aligned}
$$

where $\mu_{5}=\mu_{1} /\left(2 \pi \ell_{s}\right)^{4}$ and $\Omega_{4}=8 \pi^{2} / 3$ is the area of a unit four-sphere. This is precisely the D5-source term we expect, after averaging over the angles, and the number of D5-branes is given by

$$
\frac{6 N(n+2)}{c^{3 / 2}}=\frac{(n+1)(n+2)^{2}(n+3)}{n^{3 / 2}(n+4)^{3 / 2}} \simeq n,
$$

for large $N$. Thus, in this limit, the funnel appears to expand into $n$ D5-branes. We can also now comment on the alternate signs which we introduced in the ansatz (17): With the plus sign, the funnel carries a positive charge corresponding to $n$ D5-branes. On the other hand, with the negative sign, there is a negative charge corresponding to $n$ anti-D5-branes, by our present conventions.

Now consider the energy (24) of our configuration. Using eq. (25), this expression can be put into the following form,

$$
\begin{aligned}
E & =N T_{1} \int_{0}^{\infty} d \sigma\left[1+4 R^{4} /\left(c \lambda^{2}\right)\right]^{2} \\
& =N T_{1} \int_{0}^{\infty} d \sigma+\frac{6 N}{c} T_{5} \int_{0}^{\infty} \Omega_{4} R^{4} d R+N T_{1} \int_{0}^{\infty} d R-\Delta E
\end{aligned}
$$

where $T_{5}=T_{1} /\left(2 \pi \ell_{s}\right)^{4}$. The first term in eq. (31) corresponds to the energy of $N$ semi-infinite strings stretching from $\sigma=0$ to infinity. The second term gives the energy of $6 \mathrm{~N} / \mathrm{c}$ D5-branes 
spanning the $X^{1,2,3,4,5}$ hyperplane. Note that in the large $N$ limit, $6 N / c \sim n$ and so the energy calculation reproduces the result that $n$ D5-branes appear at $\sigma=0$, as we inferred from the charge calculation above. These first two terms in eq. (31) precisely parallel the two terms making up the total energy of the supersymmetric D3 $\perp$ D1 system. The current configuration however is not supersymmetric and so there are other contributions to the energy. The third term seems to correspond to the energy of $N$ D-strings running out radially across D5-brane world volume. The last contribution is a finite binding energy:

$$
\Delta E=2 N T_{1}\left(\frac{c \lambda^{2}}{2}\right)^{1 / 4} \int_{0}^{\infty} d u u^{4}\left[1+\frac{1}{2 u^{4}}-\sqrt{1+\frac{1}{u^{4}}}\right] \approx 1.0102 N c^{1 / 4} T_{1} \ell_{s}
$$

We will further discuss this result for the energy in the last section.

The above discussion concerns only an infinite funnel solution, but let us return to the full equation of motion to look for other solutions. The equation of motion derived from the action (23) for the radial profile is

$$
\frac{1}{R^{\prime}} \frac{d}{d \sigma} \frac{1+4 R^{4} /\left(c \lambda^{2}\right)}{\sqrt{1+\left(R^{\prime}\right)^{2}}}=0
$$

This is easily integrated once to yield

$$
\left(R^{\prime}\right)^{2}=B^{2}\left(1+\frac{4 R^{4}}{c \lambda^{2}}\right)^{2}-1
$$

where $B^{2}$ is a dimensionless integration constant. Just as for the D3 $\perp$ D1 system [14], there are three classes of solutions depending on the value of $B^{2}$. If $B^{2}=1$, eq. (34) coincides with the condition (25) minimizing the energy (24). Hence, this choice reproduces the infinite funnels discussed above. For $B^{2}<1$, the solution reaches $R=0$ at finite $\sigma$ yielding a pinched off funnel. This solution is naturally continued beyond $R=0$ with another pinched funnel, and the resulting configuration would have the interpretation of two sets of parallel D5-branes joined by a finite D-string. Finally, for $B^{2}>1$, the solution reaches $R^{\prime}=0$ at finite $\sigma$ and terminates. Again by matching the appropriate solutions, we can construct a double funnel which in this case describes a D-string joining parallel D5-branes and anti-D5-branes. We do not elaborate on the details of the double funnel constructions as they are made in complete

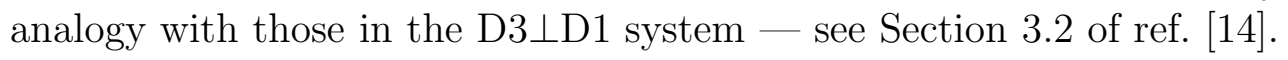

\section{D5-branes growing D-strings}

We have seen in the previous section that the D-string theory contains solutions where the D-strings expand into a set of orthogonal D5-branes. Now we want to investigate to what extent these results can be reproduced from the dual point of view of the D5-brane world volume theory. The charge conservation arguments presented in the introduction showed that 
the orthogonal D-strings act as a source of second Chern class in the world volume theory of the D5-branes. To be precise, if $N$ D-strings end on a set of D5-branes, we require

$$
\frac{1}{8 \pi^{2}} \int_{S^{4}} \operatorname{Tr} F \wedge F=N
$$

for any four-sphere surrounding the D-string endpoints. Hence we must excite a non-abelian gauge field in the D5-brane theory, which in turn means that we must consider a collection of, say, $n$ coincident D5-branes for which the world volume gauge symmetry is enhanced to $U(n)$. By analogy to the bion spike on the D3-brane, we must also excite one of the transverse scalars, say $\phi=\phi^{9}$, but it must reside in the overall $U(1)$ component of the $U(n)$ gauge symmetry to represent a collective deformation of the geometry of all the D5-branes. The relevant world volume action for the $n$ D5-branes reads

$$
S_{5}=-T_{5} \int d^{6} \sigma \operatorname{STr} \sqrt{-\operatorname{det}\left(G_{a b}+\lambda^{2} \partial_{a} \phi \partial_{b} \phi+\lambda F_{a b}\right)} .
$$

We introduce spherical coordinates on the D5-brane world volume, with radius $r$ and angles $\alpha^{i}(i=1, \ldots, 4)$. The line element may be written

$$
d s^{2}=G_{a b} d \sigma^{a} d \sigma^{b}=-d t^{2}+d r^{2}+r^{2} g_{i j} d \alpha^{i} d \alpha^{j}
$$

where $g_{i j}$ is the metric on a four-sphere with unit radius,

$$
g_{i j}=\operatorname{diag}\left[1, \sin ^{2}\left(\alpha^{1}\right), \sin ^{2}\left(\alpha^{1}\right) \sin ^{2}\left(\alpha^{2}\right), \sin ^{2}\left(\alpha^{1}\right) \sin ^{2}\left(\alpha^{2}\right) \sin ^{2}\left(\alpha^{3}\right)\right] .
$$

Now we look solutions with a "nearly spherically symmetric" ansatz: The scalar is only a function of the radius, i.e., $\phi=\phi(r)$. For the gauge field, we require that $A_{r}=0$ while the angular components are independent of $r$, i.e., , $A_{\alpha^{i}}=A_{\alpha^{i}}\left(\alpha^{j}\right)$. Examining the full equations of motion shows that this is a consistent ansatz. For the non-vanishing components of the field strength, we introduce the convenient notation: $\tilde{F}_{i j} \equiv \lambda F_{\alpha^{i} \alpha^{j}}$. Further define

$$
\tilde{F}^{i j} \equiv g^{i k} g^{j l} \tilde{F}_{k l}, \quad{ }^{*} \tilde{F}_{i j}=\frac{1}{2} \epsilon_{i j k l} \tilde{F}^{k l}
$$

i.e., the indices are raised with the inverse metric on the unit four-sphere, and the dual is defined using the volume form of the unit four-sphere, with $\epsilon_{1234}=\sqrt{g}\left(\right.$ where $\left.g \equiv \operatorname{det}\left(g_{i j}\right)\right)$. With these definitions, the charge conservation condition (35) becomes

$$
\frac{1}{16 \pi^{2} \lambda^{2}} \int \operatorname{Tr}\left(\tilde{F}_{i j}^{*} \tilde{F}^{i j}\right) \sqrt{g} d^{4} \alpha=N
$$

Substituting in the ansatz given above, the action (36) can be evaluated as

$$
S_{5}=-T_{5} \int d^{6} \sigma \sqrt{g} \sqrt{1+\lambda^{2}\left(\phi^{\prime}\right)^{2}} \mathrm{~S} \operatorname{Tr} \sqrt{r^{8}+\frac{1}{2} r^{4} \tilde{F}_{i j} \tilde{F}^{i j}+\frac{1}{16}\left(\tilde{F}_{i j}{ }^{*} \tilde{F}^{i j}\right)^{2}} .
$$


As in the previous section, the expression under the square root does not lend itself to being written as a sum of squares which would facilitate a minimum-energy analysis. We will return to this point later, but for now we study instead the equations of motion directly.

The equation of motion for $\phi$ is simply

$$
\frac{d}{d r} \frac{\partial S_{5}}{\partial \phi^{\prime}}=0
$$

which yields

$$
\frac{\lambda^{2} \phi^{\prime}}{\sqrt{1+\lambda^{2}\left(\phi^{\prime}\right)^{2}}}=\frac{f\left(\alpha^{i}\right) / \sqrt{g}}{\operatorname{STr} \sqrt{r^{8}+\frac{1}{2} r^{4} \tilde{F}_{i j} \tilde{F}^{i j}+\frac{1}{16}\left(\tilde{F}_{i j}{ }^{*} \tilde{F}^{i j}\right)^{2}}}
$$

where $f\left(\alpha^{i}\right)$ is an arbitrary function independent of $r$. Now the left hand side is a function of $r$ only, so for consistency with our ansatz, we require: $f\left(\alpha^{i}\right)=\lambda^{3} \sqrt{g} / \tilde{B}$ where $\tilde{B}$ is a dimensionless integration constant, and the gauge field must be such that the denominator above is independent of the angles.

The gauge field equation of motion can be written

$$
D_{i}\left[\sqrt{g} \frac{r^{4} \tilde{F}^{i j}+\frac{1}{4} * \tilde{F}^{i j} \tilde{F}_{k l} * \tilde{F}^{k l}}{\sqrt{r^{8}+\frac{1}{2} r^{4} \tilde{F}_{i j} \tilde{F}^{i j}+\frac{1}{16}\left(\tilde{F}_{i j} * \tilde{F}^{i j}\right)^{2}}}\right]=0
$$

It is easy to see that if we choose a self-dual field strength $\tilde{F}_{i j}={ }^{*} \tilde{F}_{i j}$, the above equation reduces to the usual Yang-Mills equations, which are then automatically satisfied due to the Bianchi identity. The scalar equation of motion (43) then imposes the further restriction that $\tilde{F}_{i j}^{*} \tilde{F}^{i j}$ should be independent of the angles, i.e., the equations of motion restrict our "nearly spherically symmetric" ansatz to a fully spherically symmetric solution. Of course, we could also choose an anti-self-dual field strength with negative instanton number (representing an anti-D-string spike), giving identical results.

For now let us assume that the desired instanton solutions exist - we will return to this point below. With $\int \sqrt{g} d^{4} \alpha=8 \pi^{2} / 3$, eq. (40) yields that

$$
\operatorname{Tr} \tilde{F}_{i j}{ }^{*} \tilde{F}^{i j}=6 N \lambda^{2} \text {. }
$$

Similarly with a self-dual gauge field, the square root that appears in various expressions above becomes

$$
\mathrm{S} \operatorname{Tr} \sqrt{r^{8}+\frac{1}{2} r^{4} \tilde{F}_{i j} \tilde{F}^{i j}+\frac{1}{16}\left(\tilde{F}_{i j}{ }^{*} \tilde{F}^{i j}\right)^{2}}=\operatorname{Tr}\left(r^{4}+\frac{1}{4} \tilde{F}_{i j}^{*} \tilde{F}^{i j}\right)=n r^{4}+\frac{3}{2} N \lambda^{2} .
$$

The equation of motion (43) for $\phi(r)$ can then be written

$$
\lambda^{2}\left(\phi^{\prime}\right)^{2}=\frac{1}{\tilde{B}^{2}\left(\frac{n r^{4}}{\lambda^{2}}+\frac{3}{2} N\right)^{2}-1} .
$$


To compare with the radial profile (34) found in the D-string description, we identify the physical transverse distance as $\sigma=\lambda \phi$, and equate the radii $r=R$. Then we see the form of the two equations agrees provided that we set $\tilde{B}=2 B / 3 N$. Complete agreement of the equations requires the coefficients $4 / c$ and $2 n /(3 N)$ to be equal, and in fact, this equality is achieved in the large $N$ limit. Hence in this limit we have complete agreement for the geometry determined by the two dual approaches! This agreement would hold for the double funnel solutions discussed below eq. (34), as well as for the infinite funnel which is the focus of our investigation.

For the spherically symmetric spike describing semi-infinite D-strings, the energy is easily evaluated to be

$$
\begin{aligned}
E & =T_{5} \int \sqrt{g} d^{4} \alpha d r \sqrt{1+\lambda^{2}\left(\phi^{\prime}\right)^{2}}\left(n r^{4}+\frac{3}{2} N \lambda^{2}\right) \\
& =N T_{1} \int d \sigma+n T_{5} \int \Omega_{4} r^{4} d r+N T_{1} \int d r-\Delta E
\end{aligned}
$$

with $\Delta E \approx 1.0102 N(6 N / n)^{1 / 4} T_{1} \ell_{s}$. Again we have full agreement with the D-string result (31) in the limit of large $N$. Note that in analogy with the D3 $\perp$ D1 analysis, the above result includes a contribution of precisely $n$ D5-branes, while the D-string calculations yield $1 / n$ corrections to the coefficient of this term.

The one point we have not yet addressed is finding an actual gauge field solution corresponding to a homogeneous instanton on the four-sphere. The ADHM construction reduces the problem of finding instanton configurations on $\mathbb{R}^{4}$ to an explicit algebraic procedure see, e.g., ref. [31]. Due to conformal invariance of the Yang-Mills system, instanton solutions on the four-sphere can then be produced with the usual stereographic projection of $S^{4}$ onto $\mathbb{R}^{4}$. For instanton number $N=1$ (or -1 ) and gauge group $S U(2)$, one finds that the unit size instanton located at the origin of $\mathbb{R}^{4}$ projects to a homogeneous instanton configuration on the four-sphere. We describe this configuration is some detail in appendix B for the interested reader. Replacing the fundamental $S U(2)$ generators by an $n \times n$ representation allows us to embed this homogeneous solution in an $S U(n)$ gauge theory. The instanton number of the resulting $S U(n)$ gauge field is maximized by choosing the irreducible representation, giving

$$
N=\frac{1}{8 \pi^{2}} \int_{S^{4}} \operatorname{Tr} F \wedge F=\frac{n\left(n^{2}-1\right)}{6} .
$$

Now a key result is that using a theorem by H.C. Wang [32], one may prove that this is the homogeneous instanton configuration on the four-sphere with the largest possible value of the second Chern class - see discussion in Appendix B. So in particular for $S U(2)$ instantons on $S^{4}$, the only $S O(5)$ invariant configurations will have $N=1,0$ or -1 . For the higher rank case of $S U(n)$, we can of course obtain an instanton number lower than in eq. (49) by choosing a reducible $n \times n$ representation of the $S U(2)$ algebra. This would correspond to several decoupled bunches of D5-branes each of which would support separate bundles of D-strings. Also, the

\footnotetext{
${ }^{1}$ We would like to acknowledge Jan Segert for invaluable assistance on this issue.
} 
scalar field $\phi(r)$ would necessarily be a more general diagonal matrix made up of a direct sum of independent $\mathrm{U}(1)$ subgroups rather than proportional to the full $n \times n$ identity matrix. In this situation the different parts of the problem completely decouple.

In fact we see from the energy (48) that the irreducible representation is preferred: As we will describe in the discussion section, for a given number $N$ of D-strings and an unlimited supply of D5-branes, we maximize $\Delta E$ (and thus minimize the energy) by coupling the $\mathrm{D}$-strings as a single bundle to a number of D5-branes given by eq. (49).

Note that for large $n$, the upper bound (49) on $N$ agrees exactly with the mysterious restriction (18) that appears in the construction of the fuzzy funnel! While this agreement is a remarkable success of the duality between the D-string and D5-brane descriptions, it appears that this restriction arises from our use of a spherically symmetric ansatz. Presumably the restriction can be evaded if we were to consider more general field configurations, although as a practical matter this would make the analysis much more difficult.

With a fully spherically symmetric ansatz as required by the equations of motion, we can also perform a minimum-energy analysis in analogy with the D3 $\perp$ D1-brane case. Beginning with D5-brane action (41), the energy may be suggestively written as

$$
\begin{aligned}
E= & T_{5} \int \sqrt{g} d^{4} \alpha r^{4} d r \operatorname{STr}\left[\left(\sqrt{1+\frac{1}{2} r^{-4} \tilde{F}^{*} \tilde{F}} \lambda \phi^{\prime} \mp \frac{1}{4} r^{-4} \tilde{F}^{*} \tilde{F}\right)^{2}+\right. \\
& \left.+\frac{1}{4} r^{-4}\left(\tilde{F}-{ }^{*} \tilde{F}\right)^{2}\left(1+\lambda^{2}\left(\phi^{\prime}\right)^{2}\right)+\left(\sqrt{1+\frac{1}{2} r^{-4} \tilde{F}^{*} \tilde{F}} \pm \frac{1}{4} r^{-4} \tilde{F}^{*} \tilde{F} \lambda \phi^{\prime}\right)^{2}\right]^{1 / 2} \\
\geq & T_{5} \int \sqrt{g} d^{4} \alpha r^{4} d r \operatorname{Tr}\left[\sqrt{1+\frac{1}{2} r^{-4} \tilde{F}^{*} \tilde{F}} \pm \frac{1}{4} r^{-4} \tilde{F}^{*} \tilde{F} \lambda \phi^{\prime}\right],
\end{aligned}
$$

where $\tilde{F}^{*} \tilde{F} \equiv \tilde{F}_{i j}{ }^{*} \tilde{F}^{i j}$. The lower bound on the energy is achieved 2 with $\tilde{F}_{i j}={ }^{*} \tilde{F}_{i j}$ and

$$
\lambda \phi^{\prime}= \pm \frac{1}{4} r^{-4} \tilde{F}^{*} \tilde{F} / \sqrt{1+\frac{1}{2} r^{-4} \tilde{F}^{*} \tilde{F}} .
$$

Now substituting in eq. (45), eq. (51) coincides precisely with the equation governing the profile of the infinite spike, i.e., eq. (47) with $\tilde{B}=2 / 3 N$. Claiming that the lower bound is a true minimum energy is not quite rigorous, as the lower bound is not just a sum of "trivial" and "topological" terms, as in the D3 $\perp$ D1-brane case. Combining spherical symmetry and the charge quantization condition (40), one has that $\tilde{F}^{*} \tilde{F}$ is independent of $r$, and so the second term is indeed topological. Note that the "trivial" term contains the contributions of the D5-branes, the radial D-strings and the binding energy.

Note that for eq. (51) to have a sensible interpretation, all of the gauge covariant expressions are understood to be proportional to the identity matrix. This is achieved by selecting the irreducible $S U(2)$ representation in the construction of the instanton as described above. For the reducible cases, the equation (50) decouples into separate parts after extending $\phi(r)$ to a more general diagonal matrix, again as discussed above.

\footnotetext{
${ }^{2}$ There is a similar bound for anti-self-dual field strengths, obtained by flipping the sign of ${ }^{*} \tilde{F}$ everywhere.
} 


\section{Generalization to dyonic strings}

It is straightforward to extend the discussion to dyonic strings [10, 33, 34, rather than Dstrings, ending on D5-branes. That is, replace the D-strings above with $\left(N, N_{f}\right)$-strings, i.e., bound states of $N$ D-strings and $N_{f}$ fundamental strings. The revised analysis is extended in complete analogy with that for the D3 $\perp$ D1 system - see, e.g., refs. [14, 25] - and so we present only the salient calculations.

Let us start with the D-string world volume theory, where fundamental strings are introduced by adding a $U(1)$ electric field. Denoting the electric field as $F_{\tau \sigma}=\mathcal{E} \mathbf{1}_{N}$, the D-string action (23) becomes

$$
S=-N T_{1} \int d^{2} \sigma \sqrt{1-\lambda^{2} \mathcal{E}^{2}+\left(R^{\prime}\right)^{2}}\left[1+4 R^{4} /\left(c \lambda^{2}\right)\right]
$$

Now the gauge field equations of motion set $\mathcal{E}$ to be a constant. The latter is then fixed by the quantization condition on the displacement, $D=N_{f} / N$, where

$$
D \equiv \frac{1}{N} \frac{\delta S}{\delta \mathcal{E}}=\frac{\lambda^{2} T_{1} \mathcal{E}}{\sqrt{1-\lambda^{2} \mathcal{E}^{2}}}
$$

after using the equations of motion. We note that rescaling $\sigma$ to $\hat{\sigma}=\sqrt{1-\lambda^{2} \mathcal{E}^{2}} \sigma$ in eq. (52) precisely reproduces the action (23) with no electric field. Hence the modified profile, in the presence of the $N_{f}$ fundamental strings, is just a stretched out version of the previous result. To be precise, we have

$$
\sigma_{\left(N, N_{f}\right)}(R)=\sqrt{1+\left(g_{s} N_{f} / N\right)^{2}} \sigma_{(N, 0)}(R)
$$

using $T_{1}=\left(\lambda g_{s}\right)^{-1}$ where $g_{s}$ is the string coupling. Hence for large $N_{f}$, the profile keeps behaving like the harmonic solution further out from the D5-brane, as would be expected for a collection of fundamental strings — see the discussion section. To calculate the energy, we evaluate the Hamiltonian, $E=\int d \sigma(D \mathcal{E}-\mathcal{L})$. The only difference in the final result compared to eq. (31), is that the contribution involving the perpendicular strings becomes

$$
E \simeq \sqrt{N^{2}+g_{s}^{2} N_{f}^{2}} T_{1} \int_{0}^{\infty} d \sigma,
$$

as expected for the dyonic strings. The energy of the radial D-strings, however, is unchanged, in accord with expectations.

From the point of view of the D5-brane world volume theory, the fundamental strings act as electric point charges [6]. Hence in this case, we add a static radial electric field $\mathcal{E}(r)=$ $F_{0 r}(r)=-A_{0}^{\prime}(r)$ in the $U(1)$ sector of the D5-brane theory. The only change in the D5-brane action (41) is that $\left(\phi^{\prime}\right)^{2} \rightarrow\left(\phi^{\prime}\right)^{2}-\left(A_{0}^{\prime}\right)^{2}$, which means that $\phi(r)$ and $A_{0}(r)$ will have the same equations of motion. Therefore we will find solutions with $A_{0}(r)=\alpha \phi(r)$, where $\alpha$ a constant. 
In terms of the rescaled scalar, $\hat{\phi}(r)=\sqrt{1-\alpha^{2}} \phi(r)$ the equations and the solution are exactly as before. Again the only modification to the energy is that the contribution representing the perpendicular strings is multiplied by a factor of $1 / \sqrt{1-\alpha^{2}}$. We can use this result to read off the value of $\alpha$, namely,

$$
\alpha=\frac{g_{s} N_{f}}{\sqrt{N^{2}+g_{s}^{2} N_{f}^{2}}},
$$

completing the specification the solution. This constant can also be fixed to the same value by charge quantization arguments. In any event, this value of $\alpha$ produces precisely the same stretching of the radial profile as was found in the D-string analysis (54). Notice that the electric field associated with the fundamental strings is not just a simple Coloumb field, rather it has the structure of the profile function $\phi^{\prime}(r)$. For large $g_{s} N_{f}$, however, the region where the field is approximately a Coloumb field becomes large. Further in this limit, the field agrees precisely with that expected for an fundamental-string spike (78).

\section{$6 \quad$ Linearized fluctuations}

For the D3 $\perp$ D1 system, the dynamics of the both the bion spike [6, 26] and the fuzzy funnel [14] were studied by considering linearized fluctuations around the static solutions presented in Section 2. Given the fluctuation equations, two interesting comparisons were made. First, one can compare the propagation of perturbations on the D3-brane spike with that on the D-string funnel. Here, at least for large $N$ and low angular momenta (on the two-sphere crosssections), there was good agreement between the two descriptions. As explained in ref. [14], producing agreement for high angular momentum would require an analysis which goes beyond the low energy Born-Infeld action. A second interesting comparison was made to perturbations propagating on a test string in the supergravity background of an orthogonal D3-brane [26]. One finds that the fluctuation equations for the bion correspond precisely to the wave equations for the test string, including the modifications due to the curved space time background. From a modern point of view, one can attribute [35] the success of the latter agreement as due to the AdS/CFT correspondence [36].

In the following, we begin an examination of propagation of fluctuations on the fuzzy funnel presented in Section 3. Our analysis is incomplete in that we do not present a complete understanding of how our results should be mapped to either those for a D5-brane bion spike or a test D-string in a supergravity background.

When considering fluctuations of the D5 $\perp$ D1 system, the setup similar to the D3 $\perp$ D1-case [26, 14]. There are two basic types of funnel fluctuations, the overall transverse modes in the directions perpendicular to both the D5-branes and the D-strings (i.e., $X^{6,7,8}$ ), and the relative transverse modes which are transverse to the D-strings, but parallel to the D5-brane world volume (i.e., along $X^{1,2,3,4,5}$ ). For simplicity in the following, we will only present examples of the overall transverse fluctuations. 


\section{Modes on the fuzzy four-sphere:}

For the moment, consider the general case of D-strings expanding into a $\mathrm{D}(2 k+1)$-brane. The associated funnel would naturally have the topology of $R \times S^{2 k}$. The perturbations of this geometry are naturally decomposed in terms of spherical harmonics on the $2 k$-sphere in particular this seems natural if one is to compare to the fluctuations of the dual bion spike in the $\mathrm{D}(2 k+1)$-brane description. The latter can be thought of as symmetric traceless tensor representations of the $S O(2 k+1)$ symmetry acting on the $S^{2 k}$. To be precise, given Cartesian coordinates $X^{i}$ in a $(2 k+1)$-dimensional embedding space, the $2 k$-sphere can be described as

$$
\sum_{i}\left(X^{i}\right)^{2}=c
$$

With this restriction, the spherical harmonics may then be written as

$$
a_{i_{1} i_{2} \cdots i_{\ell}} X^{i_{1}} X^{i_{2}} \cdots X^{i_{\ell}}
$$

where the $a$ are naturally symmetric, because of the commuting nature of the coordinates, and traceless, because of the restriction (57). In the construction of a fuzzy sphere, one replaces the embedding coordinates $X^{i}$ by finite dimensional matrices $G^{i}$ satisfying

$$
\sum_{i}\left(G^{i}\right)^{2}=c \mathbf{1}
$$

The spherical harmonics are then replaced by

$$
a_{i_{1} i_{2} \cdots i_{\ell}} G^{i_{1}} G^{i_{2}} \cdots G^{i_{\ell}}
$$

with the $a$ defined as above. These matrix harmonics can be regarded as yielding a modification of the algebra of the functions on the sphere, similar to usual discussions of noncommutative geometry [37. However, since the products in eq. (60) will only yield a finite number of linearly independent matrices, there will be an upper bound on $\ell$. Thus the matrix construction truncates the full algebra of functions on the sphere to those with $\ell \leq \ell_{\max }$. Thus the star product on the fuzzy sphere differs from that obtained by the deformation quantization of the Poisson structure on the embedding space, i.e., the latter is defined for the space of all square integrable functions on the sphere [38, 39].

Consider the specific case of $k=1$, i.e., D-strings expanding into a D3-brane with a funnel of topology $R \times S^{2}$. The fuzzy two-sphere [29] is constructed with the $G^{i}$ chosen to correspond to the $S U(2)$ generators $\alpha^{i}$, appearing in Section 2. As discussed there, for the irreducible $N \times N$ representation, these matrices satisfy eq. (59) with $c=N^{2}-1$. Because the $\alpha^{i}$ form a Lie algebra (12), the symmetrized products (60) with $\ell \leq N-1$ yield all of the possible independent matrix products. Hence there is a precise correspondence between these matrix expressions (60) and the spherical harmonics on $S^{2}$ up to $\ell_{\max }=N-1$.

The construction of the fuzzy four-sphere [24, 30], which corresponds to $k=2$ above, appears very similar, but in fact it yields a very different object. Here the appropriate $G^{i}$ are 
the $N \times N$ matrices described in appendix A. With these, one can construct matrix harmonics (60) with $\ell \leq \ell_{\max }=n$ [30]. However, a key difference from the fuzzy two-sphere is that here the $G^{i}$ do not form a Lie algebra, and as a result the algebra of these matrix harmonics does not close [24]! This can be understood by noting that while the $G^{i}$ alone do not form a Lie algebra, the combination of the $G^{i}$ and $G^{i j}$ do, giving a representation of the algebra $S O(1,5)$. This can be seen from the definition of the $G^{i j} \equiv\left[G^{i}, G^{j}\right] / 2$ and the commutators in eq. (86). Hence a closed algebra of matrix functions would be given by

$$
\tilde{a}_{a_{1} a_{2} \cdots a_{\ell}} \tilde{G}^{a_{1}} \tilde{G}^{a_{2}} \cdots \tilde{G}^{a_{\ell}}
$$

where the $\tilde{G}^{a}$ are generators of $S O(1,5)$ with $a=1, \ldots, 15$, and the $\tilde{a}$ are naturally symmetric in the $S O(1,5)$ indices. There are also a number of trace constraints arising from the matrix identities given in eqs. (85,87,88). Having identified $\tilde{G}^{a}=G^{a}$ for $a=1 \ldots 5$, the desired matrix harmonics would correspond to the subset of $\tilde{a}$ with nonvanishing entries only for indices $a_{i} \leq 5$. Thus while the fuzzy four-sphere construction introduces an algebra that contains a truncated set of the spherical harmonics on $S^{4}$, the algebra also contains a large number of elements transforming under other representations of the $S O(5)$ symmetry group that acts on the foursphere. We refer the reader to ref. [30] for a precise description of the complete algebra in terms of representations of $S O(5)$ (or rather $\operatorname{Spin}(5)=S p(4)$ ).

When it comes to the physical fluctuations of the fuzzy funnel describing D-strings expanding into D5-branes, we stress that both sets of these modes play a role. For the purposes of illustration, it is sufficient to only consider the lowest order equations of motion (21) and examine linearized fluctuations $\delta \Phi^{m}$. The linearized equation is

$$
\left(-\partial_{\tau}^{2}+\partial_{\sigma}^{2}\right) \delta \Phi^{m}=\left[\Phi^{j},\left[\Phi^{j}, \delta \Phi^{m}\right]\right]+\left[\Phi^{j},\left[\delta \Phi^{j}, \Phi^{m}\right]\right]+\left[\delta \Phi^{j},\left[\Phi^{j}, \Phi^{m}\right]\right] .
$$

As mentioned above, we will focus on overall transverse fluctuations involving, e.g., $\delta \Phi^{6}$. First consider the matrix harmonic modes (60), i.e., a fluctuation proportional to a symmetric traceless product of $G^{i}$ s,

$$
\delta \Phi^{6}=a_{i_{1} i_{2} \cdots i_{l}} G^{i_{1}} G^{i_{2}} \cdots G^{i_{l}} .
$$

The lowest order equation (62) then becomes

$$
\left[\partial_{\tau}^{2}-\partial_{\sigma}^{2}+\frac{l(l+3)}{2 \sigma^{2}}\right] a_{i_{1} \cdots i_{l}}(\sigma, \tau)=0 .
$$

Notice that the double commutator terms on the right hand side of eq. (62) have produced the correct angular momentum barrier for a spherical harmonic mode propagating on the $S^{4}$.

As discussed earlier, however, we need also consider more general fluctuations which include the $G^{i j}$. The simplest example is given by, $\delta \Phi^{6}=\tilde{a}_{i j} G^{i j}$ where $\tilde{a}_{i j}=-\tilde{a}_{j i}$. Substituting into the equation of motion (62) yields

$$
\left[\partial_{\tau}^{2}-\partial_{\sigma}^{2}+\frac{1}{\sigma^{2}}\right] \tilde{a}_{i j}(\sigma, \tau)=0 .
$$


Hence the equation of motion has essentially the same form as for the matrix harmonics. This illustrates then that within the context of the fuzzy funnel, the fluctuations involving $G^{i j}$ are equally valid physical modes.

If we were to compare the above fluctuations to those in the D5-brane description of the intersection, it would seem that the $\delta \Phi^{6}$ fluctuations proportional to symmetric traceless products of $G^{i}$ must correspond to $U(1)$ scalar field fluctuations of $\phi^{6}$ proportional the spherical harmonics on the (commutative) four-sphere. Identifying the D5-brane modes corresponding to $\delta \Phi^{6}$ fluctuations involving $G^{i j}$ is more puzzling. However, it seems that they must be related to non-abelian excitations of the D5-brane theory. It would be interesting to investigate this correspondence in detail, as it may yield new insights on thinking about the fuzzy four-sphere geometry. Beyond the linearized equations of motion, clues as to the interpretation of these modes can be found by investigating the nontrivial couplings which they induce. For example, the Wess-Zumino part of the non-abelian D-string action contains a term [9]:

$$
-i \frac{\lambda \mu_{1}}{3} \int d \sigma d \tau \operatorname{Tr}\left(\Phi^{i} \Phi^{j} \Phi^{k}\right) F_{\tau \sigma i j k}^{(5)}
$$

corresponding to a D3-brane dipole coupling. Hence when the antisymmetric mode considered above propagates on the fuzzy funnel, one finds

$$
-i \lambda \mu_{1} \int d \sigma d \tau \operatorname{Tr}\left(\Phi^{i} \Phi^{j} \delta \Phi^{6}\right) F_{\tau \sigma i j 6}^{(5)}=i \frac{4 \mu_{1}}{5 c \lambda} \int d \sigma d \tau R^{2}(\sigma) \tilde{a}_{i j} F_{\tau \sigma i j 6}^{(5)} .
$$

using the background $\Phi^{i}=R(\sigma) G^{i} / \sqrt{c} \lambda$. We therefore see that perturbing by these modes induces a D3-brane dipole along the fuzzy funnel. This coupling would vanish for any of the usual matrix harmonics given in eq. (63).

\section{Supergravity comparison:}

For the D3 $\perp$ D1 system, it was found that the linearized equations for the full Born-Infeld action encoded some of the modifications of the supergravity background generated by the D3-branes [26, 14]. Here we consider the analogous analysis for the fuzzy funnel describing the D5 $\perp$ D1 system.

The simplest modes are those which do not excite internal modes on the $S^{4}$, i.e., fluctuations proportional to the identity matrix, say

$$
\delta \Phi^{6}(\sigma, \tau)=f(\sigma, \tau) \mathbf{1}_{N} .
$$

Plugging this fluctuation into the full D-string action, together with the funnel ansatz $\Phi^{i}=$ $R G^{i} / \sqrt{c} \lambda$, we find

$$
\begin{aligned}
S & =-N T_{1} \int d^{2} \sigma \sqrt{\left(1+R^{\prime 2}\right)\left(1-\lambda^{2} \dot{f}^{2}\right)+\lambda^{2} f^{\prime 2}}\left[1+4 R^{4} /\left(c \lambda^{2}\right)\right] \\
& =-N T_{1} \int d^{2} \sigma H \sqrt{1-\lambda^{2} \dot{f}^{2}+\frac{\lambda^{2}}{H} f^{\prime 2}} \\
& =-N T_{1} \int d^{2} \sigma\left(H-\frac{1}{2} \lambda^{2} H \dot{f}^{2}+\frac{1}{2} \lambda^{2} f^{\prime 2}+\ldots\right)
\end{aligned}
$$


with

$$
H(\sigma)=\left(1+\frac{4 R(\sigma)^{4}}{c \lambda^{2}}\right)^{2} .
$$

In the second line above, we used the equation of motion for $R(\sigma)$, while in the third line retained only quadratic terms in $f$, as is appropriate to determine the linearized equations of motion. The latter become

$$
\left(H \partial_{\tau}^{2}-\partial_{\sigma}^{2}\right) f(\sigma, \tau)=0
$$

Evaluating this result for large $\sigma$, using the asymptotics (26), yields

$$
\left(\left[1+\frac{n^{2} \lambda^{2}}{8 \sigma^{4}}\right] \partial_{\tau}^{2}-\partial_{\sigma}^{2}\right) f(\sigma, \tau) \simeq 0 .
$$

Here we have also used $c \simeq n^{2}$ for large $n$.

Now we wish to compare this result with the fluctuation equation for a test D-string in some supergravity background, e.g., a D5-brane background. Assuming a diagonal background metric $G_{\mu \nu}$, the test D-string action becomes

$$
\begin{aligned}
\tilde{S} & =-N T_{1} \int d^{2} \sigma e^{-\phi} \sqrt{G_{\tau \tau} G_{\sigma \sigma}-\lambda^{2} G_{\sigma \sigma} G_{66} \dot{f}^{2}+\lambda^{2} G_{\tau \tau} G_{66} f^{\prime 2}} \\
& =-N T_{1} \int d^{2} \sigma e^{-\phi} \sqrt{G_{\tau \tau} G_{\sigma \sigma}}\left(1-\frac{1}{2} \lambda^{2} \frac{G_{66}}{G_{\tau \tau}} \dot{f}^{2}+\frac{1}{2} \lambda^{2} \frac{G_{66}}{G_{\sigma \sigma}} f^{\prime 2}\right) \\
& =-N T_{1} \int d^{2} \sigma\left(\sqrt{h}-\frac{1}{2} \lambda^{2} h^{3 / 2} \dot{f}^{2}+\frac{1}{2} \lambda^{2} \sqrt{h} f^{\prime 2}\right) .
\end{aligned}
$$

The second line above holds for arbitrary (diagonal) metrics, while the third line is evaluated for the metric of $n$ D5-branes,

$$
\begin{aligned}
d s^{2} & =h(\sigma)^{-1 / 2} \eta_{\mu \nu} d x^{\mu} d x^{\nu}+h(\sigma)^{1 / 2}\left(d \sigma^{2}+\sigma^{2} d \Omega_{3}^{2}\right) \\
e^{-2 \phi} & =h(\sigma) \\
h(\sigma) & =1+L^{2} / \sigma^{2}, \quad L^{2}=n \alpha^{\prime} g_{s}
\end{aligned}
$$

Note that the background RR field and the Wess-Zumino interactions do not contribute to the quadratic action in this case. Hence the the linearized equation of motion for $f$ is

$$
\left(h \partial_{\tau}^{2}-\partial_{\sigma}^{2}+\frac{L^{2}}{h \sigma^{3}} \partial_{\sigma}\right) f(\sigma, \tau)=0
$$

which does not appear to match the previous equation of motion (71). One can improve on matching the form of the equations by changing the spatial variable, $\tilde{\sigma}^{2}=\sigma^{2}+L^{2}$, which yields

$$
\left(h^{2} \partial_{\tau}^{2}-\partial_{\tilde{\sigma}}^{2}\right) f(\tilde{\sigma}, \tau)=0 .
$$

However there is still a mismatch because the 'harmonic' functions multiplying $\partial_{\tau}^{2}$ fails to agree. 
The $1 / \sigma^{4}$ terms in eq. (72) would be more appropriate for a 3 -brane-like background. This is perhaps not surprising as the large $\sigma$ behavior of the D-string funnel is universal, and will thus agree with what we found for D3 $\perp \mathrm{D} 1$. The disagreement is still puzzling, however, as we would expect both the D-string and the test-string descriptions to be valid for large $\sigma$. If we insist that the quadratic action (69) should be exactly reproduced by a supergravity background, the latter must satisfy

$$
G_{\tau \tau}=G_{66}=H^{-1} G_{\sigma \sigma}=H^{1 / 2} e^{\phi} .
$$

These precise relations need only apply along the radial line where the D-string is placed. It would be very interesting to find a physical interpretation of such a background.

Note that for the F-string spikes discussed in ref. [26], agreement with supergravity was only found for D3- and D4-branes. In the case of D5-branes one can argue that the bion description of the F-string spike has broken down at large $\sigma$. In contrast, we expect that large $\sigma$ is precisely the regime where the fuzzy funnel description of the D $5 \perp \mathrm{D} 1$ system is reliable.

\section{Discussion}

We have described two dual descriptions of the D5 $\perp$ D1 system. In particular, we have found solutions of both the low energy D1-brane and D5-brane theories describing a set of $N$ D1branes ending on a collection of $n$ orthogonal D5-branes. Good agreement was found in the formulae describing the energy, RR couplings and the funnel geometry in the two theories. While one may regard the two different types of D-branes as giving dual descriptions of the same intersection, we stress that our analysis is limited to the low energy world volume theory in each case. Hence in neither case do we have a complete description as in both theories the configurations of interest contain singularities. Our analysis therefore yields two complementary descriptions of the D $5 \perp \mathrm{D} 1$ intersection. In the region far away from the D5-branes, the fuzzy funnel construction of the D1-brane theory is reliable, while near the D5-brane, the D5-brane bion picture is trustworthy.

\section{Regimes of validity:}

One is left then with the question of why do these two descriptions agree so well for large $n$ or $N$. A similar agreement exists for the D3 $\perp$ D1 system, and in that case a careful analysis of the two complementary theories showed that for large $N$ there is a large region of overlap in which both theories give a reliable description of the intersection [14]. A similar result is found upon examining the regimes of validity of the D-string and D5-brane theories in the present case. First, we must determine when we can confidently ignore the higher derivative corrections to the Born-Infeld action arising from the usual $\alpha^{\prime}$ expansion of low energy string theory. Schematically we require that $\ell_{s}\left|\partial^{2} \Phi\right| \ll|\partial \Phi|$. For the spike solution on the D5-brane, this translates into $r^{2} \gg \ell_{s}^{2}$, while for the fuzzy funnel in the D-string theory, we find that this restriction is equivalent to $r^{2} \ll c \ell_{s}^{2}$. So for large $n$ with $c \simeq n^{2}$, there is a significant overlap region where the higher derivatives are negligible in both theories and we might expect good 
agreement.

As well as higher derivative corrections, the non-abelian action with the symmetric trace prescription 27] requires additional higher order commutator corrections [40, 41, 42] — see also the discussion in ref. [9]. For the fuzzy funnel, we might require that $\ell_{s}^{2}\left|\hat{R}^{\prime}\right| \ll 1$ or $\ell_{s}^{2} \hat{R}^{2} \ll 1$ since these dimensionless quantities characterize the relevant scalar field commutators. In terms of the physical radius, both of these restrictions can be translated into $r^{2} \ll c \ell_{s}^{2}$, which coincides with that found above. A more conservative bound for avoiding higher commutator corrections is demanding that the Taylor expansion of the square root in the action (15) should converge rapidly. The latter produces the more restrictive condition that $r^{2} \ll c^{1 / 2} \ell_{s}^{2}$. However, for large $n$, this still seems to leave room for a significant region of overlap with the D5-brane description.

The D5-brane theory is also non-abelian, and so one might consider the effect of higher commutator corrections to the spike solution as well. However, we argue that in fact these additional commutators do not affect the configuration studied here. First, as can be seen in eq. (41), the determinant in the five-brane action (36) factors into two separate terms: a radial term involving the abelian scalar, and an angular term involving the non-abelian gauge field. Hence any higher commutators will only affect the latter contribution. However, here we note that the gauge field configuration of interest is self-dual (or anti-self-dual), and investigations of the non-abelian Born-Infeld theory indicate that the higher commutator corrections do not affect such configurations. In particular, one finds that the spectrum of the non-abelian BornInfeld theory precisely matches that of the full string theory when considering excitations around a self-dual field configuration [40, 41, 42]. Hence it would appear that higher order commutators will not modify the analysis in the D5-brane theory.

Hence from our analysis, we are led to conclude that in the regime $\ell_{s}^{2} \ll r^{2} \ll n \ell_{s}^{2}$ both the D-string and D5-brane descriptions should be reliable. Thus for large $n$, we have a large region of overlap and we should expect good agreement between the D5-brane spike and the D-string's fuzzy funnel. In Section 3, the calculations are also expected to have $1 / c$ corrections since we did not implement the symmetrization of the gauge trace. However, these corrections to the D-string analysis will be negligible as long as $n$ is large. This point was not a problem in the D5-brane analysis since for (anti-) self dual gauge fields the square root in the action simplifies, as shown in eq. (46). In passing, we also point out that our calculations neglect gravitational effects, which is justified when $g_{s} N \ll 1$. However, we can simply consider very weak string coupling since $g_{s}$ does not appear in our calculations.

\section{Geometric profile:}

The full nonlinear form of the Born-Infeld action was important to produce a funnel which made a transition between the universal behavior $\left(R \sim \sigma^{-1}\right)$ at small $R$ and the harmonic behavior $\left(R \sim \sigma^{-1 / 3}\right)$ at large $R$, as discussed in Section 3. Of course, the same is true for the profile of the bion spikes constructed in Section 4 .

It is interesting to compare the profile of the D5 $\perp \mathrm{D} 1$ intersection to that for the $\mathrm{D} 5 \perp \mathrm{F} 1$ intersection. Fundamental strings ending on an orthogonal D5-brane act like electric point 
sources in the world volume theory of the D5-brane, and the intersection was studied from this point of view in ref. [6]. For $N_{f}$ fundamental strings ending on $n$ D5-branes, the transverse scalar on the D5-brane is given by

$$
\sigma(r)=\frac{N_{f} g_{s} \lambda^{2}}{2 n r^{3}}
$$

and the corresponding radial electric field on the D5-brane world volume is simply $E_{r}=\partial_{r} \sigma(r)$. This agrees with the profile of the D-string spike for small $\sigma$ (i.e., in the harmonic function regime) with the substitution $N \rightarrow N_{f} g_{s}$. However, for large $\sigma$, the D-string spike is much narrower because of the onset of the universal behavior. The two profiles are plotted together in Fig. 1.

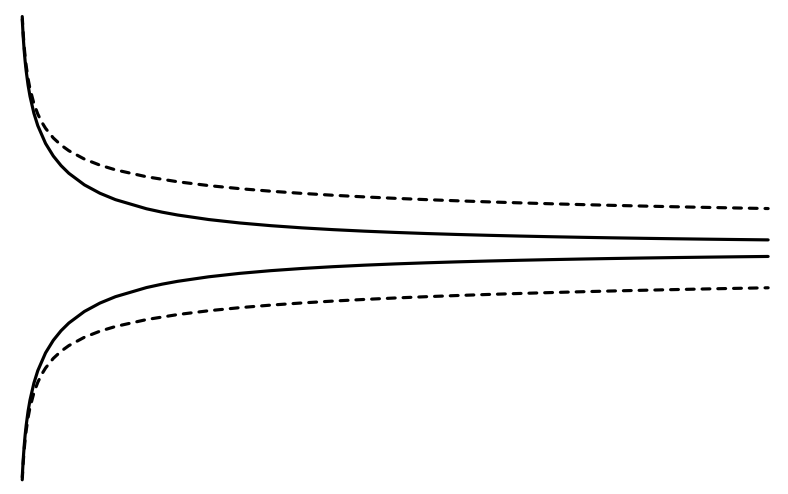

Figure 1: The shape of D-string (solid curve) and fundamental string (dashed curve) spikes extending from a set of orthogonal D5-branes. We have set $N=N_{f} g_{s}$ so that the profiles overlap for small $\sigma$.

S-duality will transform the D $5 \perp$ F1 intersection into an NS5 $\perp$ D1 intersection. Since $g_{s} \ell_{s}^{4} \sim$ $\ell_{p}^{4}$ is invariant under S-duality, eq. (78) also describes the profile for the latter system of intersecting branes. Of course, this observation begs the question of how does one realize this intersection with a fuzzy funnel description? The latter is related to the unresolved problem of constructing transverse five-branes in Matrix theory [0, 43]. For the profile $(\sqrt{78})$ above to be reliable in the D-string theory, it would have to give a macroscopic radius in a regime where the variation of the radius is still small (i.e., $r \gg \ell_{s}, \partial_{\sigma} r \ll 1$ ). Combining these constraints requires that $N g_{s} \gg 1$, which would be consistent with the idea that the D-brane couplings to the NS5-brane charge arise as a strong coupling effect.

If would also be interesting to investigate whether one can find funnel solutions for fundamental strings, giving a dual description of the $\mathrm{D} p \perp \mathrm{F} 1$ intersections. A possible framework for such an analysis is provided by matrix strings [44, 45].

Another important aspect of the geometry in our constructions was the spherical symmetry. In Section 4, this symmetry appeared essentially as an assumption which we imposed in 
order to make the problem tractable. Solving for symmetric instanton configurations yields the curious relation between $N$, the number of D-strings and $n$, the number of D5-branes: $N \simeq n^{3} / 6$ for large $N$. Remarkably, precisely the same relation arises (again for large $N$ ) from the noncommutative geometry in the construction of the D-string funnel - see eq. (18). Implicitly spherical symmetry is again an underlying assumption in the latter construction, i.e., by definition the fuzzy four-sphere is $S O(5)$ symmetric [24].

We would expect that for any $N, n \gg 1$ that the $\mathrm{D} 5 \perp \mathrm{D} 1$ intersection has a smooth geometric resolution. However, it seems that the intricacies of the non-abelian world volume theories will not allow for spherically symmetric solutions in general. This is similar to the case of BPS $S U(2)$ magnetic monopoles, which describe D-strings extending between parallel D3-branes at finite separation [46]. It is known that there are no spherically symmetric solutions for monopole charge greater than one. The best one can do is to obtain axially symmetric configurations 47]. On the D5-brane side, relaxing the condition of spherical symmetry would enable one to obtain higher instanton number solutions, but, of course, allowing for angular variations in the scalar and gauge fields makes finding solutions significantly harder. Similarly, on the D1-brane side relaxing the $S O(5)$ symmetry presumably allows for the use of more general matrices, but it is not at all clear how one should proceed if the matrices $G^{i}$ are abandoned. We leave these issues for future work.

\section{SUSY and stability:}

There is, of course, one major distinction between the $\mathrm{D} 5 \perp \mathrm{D} 1$ system and the $\mathrm{D} 3 \perp \mathrm{D} 1$ system. That is, while the latter is supersymmetric, the former is not. This is seen easily in perturbative string theory [2, 3], where one finds that having six Neumann-Dirichlet directions for the D1-D5 strings is incompatible with supersymmetric ground state. Given the lack of supersymmetry, one might worry that the configurations describing the $\mathrm{D} 5 \perp \mathrm{D} 1$ intersection are unstable. However, the inequality in eq. (24) shows that the semi-infinite fuzzy funnel is a minimum energy configuration, given the boundary conditions that have been imposed. A similar inequality (50) appeared in the discussion of the D5-brane spike. Here again we comment that in both of world volume theories, the configurations describing the D $\perp \perp \mathrm{D} 1$ intersection are singular but the singularities have an interesting physical interpretation in terms of D-brane geometries. Now once we admit such non-standard or singular boundary conditions, there is no obvious way in which the system could decay compatible with the physics of D-branes. In other words, the boundary conditions define a certain super-selection sector, and since we have the semi-infinite funnel to be the lowest energy configuration within this sector, we can expect stability, at least to perturbative deformations of the system.

We note that there does exist a supersymmetric configuration of a D1-brane orthogonal to a D5-brane where appropriate fluxes of the NS two-form field are introduced on the D5-brane [48. In fact by tuning these fluxes the D1- and D5-branes can remain supersymmetric while at any angle. The supersymmetry in these configurations is maintained by the fact that the supersymmetric variation of the D5-brane world volume gaugino is altered in the presence of the constant background B-field in such a way as to be compatible with the supersymmetry of 
the D1-brane. These results would apply most directly to an infinite D1-brane which "pierces" an orthogonal D5-brane. To go from this system to the D5 $\perp$ D1 intersection considered here, one would have to split the D-string on the D5-brane and move one half off to infinity along the D5-brane. However, it is not obvious whether or not supersymmetry could be maintained in this operation, but it would be an interesting project for future research. One final observation is that the supersymmetric configuration would not be spherically symmetric as the B-field fluxes distinguish the different directions on the D5-brane world volume.

\section{Energy:}

Being a non-supersymmetric state, the energy of the D5 $\perp$ D1 intersection is not simply the sum of the energy of the constituents. Perhaps surprisingly, the energy given in eqs. (31) or (48) splits rather nicely into four distinct parts. The first and second contributions correspond to the energy of the $n$ D5-branes and the $N$ semi-infinite D-strings extending orthogonally away from the D5-branes. Next there is a term that equals the energy of $N$ semi-infinite D-strings extending out radially in the D5-brane world volume (like the dashed lines in Fig. 7 (a) below). Finally there is a finite binding energy, given in eq. (32)..

How can we understand the appearance of the 'radial' D-string term in the energy? Loosely speaking the D-strings do not terminate at a fixed intersection point on the D5-brane, but rather they dissolve into the five-brane world volume and spread out to infinity in the guise of the gauge fields (35). One can easily confirm that the gauge configuration introduces the appropriate source for the RR two-form to correspond to the D-strings spreading out radially across the world volume. Of course, these comments would hold equally well in discussing the bion spike describing D3 $\perp \mathrm{D} 1$ intersection in Section 2. Why then is this not reflected in the bion energy (6)? If one considers parallel D-strings and D3-branes, a true bound state is formed [2, 3, 49] but in the limit of large D3-brane volume, the energy approaches that of the D3-branes alone. (The D-string charge is still given by integrating the vanishingly small magnetic flux across the entire D3-brane.) In contrast, parallel D-strings and D5-branes form a marginal bound state whose total energy is always given by the sum of that for each of the constituents. Hence, there remains a contribution in the energy from the radial spreading of D-strings. Of course, the 'radial' D-strings are not all parallel to each other, and so the analogy to the supersymmetric system of parallel D-strings and D5-branes is not precise.

In fact one might expect a large additional contribution to the energy due to the interaction between these 'radial' D-strings. However, the remaining term in the energy is a finite, negative contribution, $-\Delta E$. This is the only term which distinguishes between configurations in which the D-strings couple to the D5-brane as a single bundle or not. For instance, consider a situation where we have $N$ D-strings and a large number of D5-branes available for the D-strings to end on. One possibility is to split the D-strings into two bundles with $N_{1}$ and $N_{2}$ strings, each coupling to $n_{1}$ and $n_{2}$ D5-branes, where $n_{i} \sim N_{i}^{1 / 3}$ assuming large $n_{i}$ (using the irreducible representation in the construction of the $S U\left(n_{i}\right)$ instantons). Since $\Delta E \sim N(N / n)^{1 / 4}$ (see 


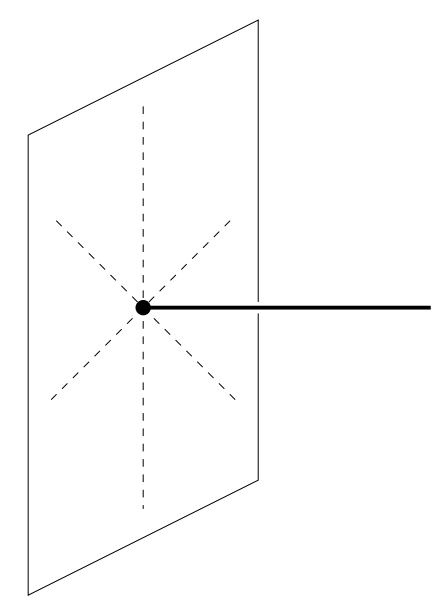

(a)

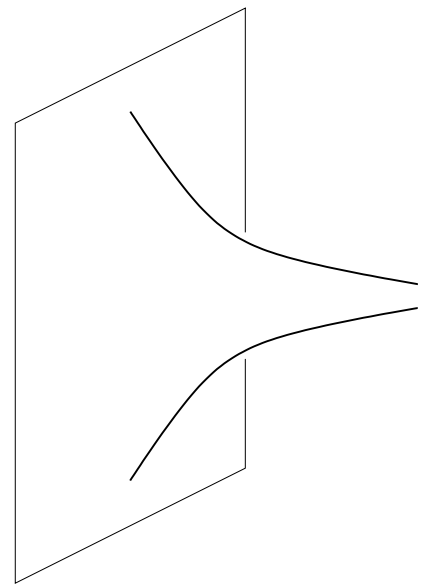

(b)

Figure 2: (a) Schematic picture of strings ending on a brane. The endpoint of the strings has a charge which is carried away by flux lines on the brane. Alternatively one can think of the flux lines as a continuation of the strings, smeared over the angular directions. (b) In reality the strings pull on the brane, creating a spiked surface.

below eq. (48)), the total binding energy for this configuration is

$$
\Delta E \sim \sum_{i} N_{i}^{7 / 6}
$$

On the other hand, all of the D-strings can be combined in a single source of instanton number. This is accomplished with roughly $N^{1 / 3}$ D5-branes, and the binding energy of such a configuration depends on $N$ as

$$
\Delta E \sim\left(N^{7 / 6}\right)=\left(\sum_{i} N_{i}\right)^{7 / 6}
$$

Hence this single bundle of D-strings has a larger binding energy than the one in eq. (79). Noting that $\Delta E$ contributes with a negative sign to the total energy, we conclude that for $N$ D-strings and a large available number of D5-branes, we minimize the energy (48) by coupling all the D-strings to the smallest possible number $n$ of D5-branes compatible with the upper bound (49).

It is also interesting to compare our results for the D $\perp \perp \mathrm{D} 1$ intersection to the supergravity picture with test D-strings in the background corresponding orthogonal D5-branes. The latter framework yields the correct energy for the D3 $\perp$ D1 system. The relevant fields for the D5-brane background are given in eq. (74). The energy of $N$ test D-strings extending along $\sigma$ in this geometry is given by

$$
E=N T_{1} \int_{0}^{\infty} d \sigma e^{-\phi} \sqrt{G_{t t} G_{\sigma \sigma}}=N T_{1} \int_{0}^{\infty} d \sigma \sqrt{1+L^{2} / \sigma^{2}}
$$


Here, of course, we find a divergent contribution as $\sigma \rightarrow \infty$ corresponding to the energy of the D-strings extending off to infinity. Note, however, that there is a logarithmic divergence in the energy down the throat of the D5-brane as $\sigma \rightarrow 0$. This divergence emerges because of the nontrivial dilaton profile in the D5-brane background (74). It is tempting to associate this divergent energy with the contribution which we identified with the radial D-strings above, but the precise form of the divergences does not seem to agree. Even the leading order deviations from flat space at large $\sigma$ disagree, similar what was found for the fluctuations. We should also note that due to the variation of the background dilaton, the effective string coupling is becoming smaller as we approach the D5-branes, and thus the effective D-string tension (i.e., energy density) is becoming large. At some point, the test brane picture of the D-strings will break down, and there will be significant corrections to the geometry. This suggests that the D5-brane supergravity background is not the relevant one for this system. In fact, the supergravity background (77) we introduced in the analysis of the fluctuation equations, also yields the correct test-string energy. This is to be expected since the relations in eq. (77) ensured that the quadratic actions agreed including the constant term. The latter essentially corresponds to the energy density. It would be interesting to address these issues in the context of the AdS/CFT along the lines of ref. [50].

\section{Other fuzzy funnels:}

In this paper, we have constructed the simplest extension of a new class of brane intersections in analogy with the D3 $\perp$ D1 system. The smooth resolution of the D $5 \perp \mathrm{D} 1$ intersection owes its existence to the non-abelian nature of the world volume gauge theories. It also provides another example of the duality by which a given brane configuration can be described by two different world volume theories. There are many other extensions of this construction that might be considered.

For instance, one might construct fuzzy funnels with other noncommutative geometries. For example, a number of different fuzzy geometries are considered in ref. [17], including the coset geometries: $S U(2) / U(1) \times S U(2) / U(1), S U(3) / U(2)$ and $S U(3) / U(1) \times U(1)$. While there the analysis focussed on the dielectric effect, their constructions can easily be adapted to produce new non-abelian brane intersections. The first case above would simply yield a fuzzy funnel with cross-section $S^{2} \times S^{2}$. Of course, the interpretation would be that of a D1-brane intersecting a pair of mutually orthogonal D3-branes. The case of $S U(3) / U(2) \sim C P^{2}$ would give a four-dimensional cross-section and one can easily verify that the funnel couples to the RR six-form. Hence this would also yield a D $\perp \perp \mathrm{D} 1$ intersection but with a more exotic five-brane geometry. Finally, the coset $\mathrm{SU}(3) / \mathrm{U}(1) \times \mathrm{U}(1)$ is six-dimensional and from the RR couplings, it appears the the corresponding funnel would describe the D-strings expanding into a bound state of D7- and D5-branes.

A less exotic intersection which would be interesting is the $\mathrm{D} 7 \perp \mathrm{D} 1$ system. In this case, the system would be supersymmetric and so perhaps the world volume field theory configurations would be more reliable. In the D7-brane theory, one would have to consider gauge field configurations on the six-sphere with nonvanishing third Chern character. For the D-string de- 
scription, the funnel would require a fuzzy $S^{6}$. It may be that these configurations are related to the work of ref. [51].

Another interesting extension of our work would be to consider D-strings joining collections of parallel D5-branes with finite separation. These configurations would be related to the double funnels mentioned in Sections 3 and 4. However, given the results for the analogous configurations of D-strings and D3-branes [46, 14, it is likely that the true minimum energy configurations will not be spherically symmetric. It would also be interesting to consider the asymmetric case where the numbers of D5-branes at either end of the D-string are not equal. This would lead to exotic funnels similar to the domain walls considered in ref. [18].

Perhaps the ideas considered here could also be extended to even more exotic intersections in M-theory, involving M2-branes, M5-branes and KK monopoles, using the non-abelian couplings proposed in ref. [20].

\section{Acknowledgments}

This research was supported by NSERC of Canada and Fonds FCAR du Québec. RCM was also partially supported by the CIAR Cosmology and Gravity Program. We would especially like to thank Kiril Krasnov and Jan Segert for helpful discussions. We would also like to acknowledge interesting conversations with Bobby Acharya, Vijay Balasubramanian, Marco Gualtieri, Nigel Hitchin, Neil Lambert, Washington Taylor and Mark Van Raamsdonk. We also thank the Institute for Theoretical Physics at UCSB for its hospitality during the early stages of this work.

\section{A The $G^{i}$ matrices}

For the convenience of the reader, we here reproduce several formulae involving the matrices $G^{i}$ which were used to construct our D-string funnel. These matrices were presented in ref. 24] to provide a fuzzy four-sphere. They satisfy certain physical properties including producing a spherical locus, $S O(5)$ rotational invariance and an appropriate spectrum of eigenvalues. The equations below are taken directly from the paper by Castelino, Lee and Taylor [24], and we refer the reader there for more details.

The matrices $G^{i}$ and $G^{i j}$ are defined as follows:

$$
\begin{aligned}
G^{i} & =\left(\Gamma^{i} \otimes \mathbf{1} \otimes \cdots \otimes \mathbf{1}+\mathbf{1} \otimes \Gamma^{i} \otimes \mathbf{1} \otimes \cdots \otimes \mathbf{1}+\cdots+\mathbf{1} \otimes \cdots \otimes \mathbf{1} \otimes \Gamma^{i}\right)_{\mathrm{Sym}}, \\
G^{i j} & =\frac{1}{2}\left[G^{i}, G^{j}\right],
\end{aligned}
$$

where $\Gamma^{i}, i=1, \ldots, 5$ are $4 \times 4$ Euclidean gamma matrices, and $\mathbf{1}$ is the identity matrix. The subscript Sym means the matrices are restricted to the completely symmetric tensor product 
space. An explicit construction of these matrices in $2 \times 2$ block-diagonal form is given by

$$
\begin{aligned}
\Gamma^{j} & =\left(\begin{array}{cc}
0 & -i \sigma_{j} \\
i \sigma_{j} & 0
\end{array}\right), \quad j \in\{1,2,3\}, \\
\Gamma^{4} & =\left(\begin{array}{cc}
0 & \mathbf{1}_{2} \\
\mathbf{1}_{2} & 0
\end{array}\right), \\
\Gamma^{5} & =\left(\begin{array}{cc}
\mathbf{1}_{2} & 0 \\
0 & -\mathbf{1}_{2}
\end{array}\right),
\end{aligned}
$$

where $\sigma_{j}$ are the usual Pauli matrices.

The dimension and the "Casimir" of the $n$-fold totally symmetric representation $G^{i}$ are

$$
N=\frac{(n+1)(n+2)(n+3)}{6}, \quad c=n(n+4) .
$$

The Casimir, $c$, appears in the following identities:

$$
G^{i} G^{i}=c \mathbf{1}_{N}, \quad G^{i j} G^{j i}=4 c \mathbf{1}_{N} .
$$

The commutators of $G^{i}$ 's and $G^{j k}$ 's are easily obtained from those of gamma matrices:

$$
\begin{aligned}
{\left[G^{i j}, G^{k}\right] } & =2\left(\delta^{j k} G^{i}-\delta^{i k} G^{j}\right), \\
{\left[G^{i j}, G^{k l}\right] } & =2\left(\delta^{j k} G^{i l}+\delta^{i l} G^{j k}-\delta^{i k} G^{j l}-\delta^{j l} G^{i k}\right) .
\end{aligned}
$$

Note then that the $G^{i k}$ are the generators of $S O(5)$ rotations. There is a very useful identity between the anti-commutators:

$$
\left\{G^{i j}, G^{j k}\right\}+\left\{G^{i}, G^{k}\right\}=2 c \delta^{i k} \mathbf{1}_{N},
$$

and we also need the following identities:

$$
\begin{aligned}
G^{i j} G^{j} & =4 G^{i}, \\
G^{i j} G^{j k} & =c \delta^{i k}+G^{i} G^{k}-2 G^{k} G^{i}, \\
\epsilon^{i j k l m} G^{i} G^{j} G^{k} G^{l} & =(8 n+16) G^{m} .
\end{aligned}
$$

Combining the above definitions and identities, it is straightforward to derive the following formulae

$$
\begin{array}{cl}
G^{i} G^{j} G^{i} G^{j}=\left(c^{2}-8 c\right) \mathbf{1}_{N}, & G^{i} G^{j} G^{i k} G^{k j}=-8 c \mathbf{1}_{N}, \\
G^{i} G^{j k} G^{i} G^{j k}=\left(-4 c^{2}+16 c\right) \mathbf{1}_{N}, & G^{i j} G^{k l} G^{i j} G^{k l}=\left(16 c^{2}-96 c\right) \mathbf{1}_{N}, \\
G^{i j} G^{j k} G^{k l} G^{l i}=\left(4 c^{2}+32 c\right) \mathbf{1}_{N}, & G^{i j} G^{j k} G^{i l} G^{l k}=\left(4 c^{2}-40 c\right) \mathbf{1}_{N}, \\
G^{i} G^{j} G^{j k} G^{k i}=G^{i} G^{i k} G^{j} G^{j k}= & G^{i} G^{j k} G^{j} G^{i k}=16 c \mathbf{1}_{N} .
\end{array}
$$




\section{B Homogeneous instantons}

It is easy to construct self-dual $S U(2)$ gauge fields which are homogeneous on $S^{4}$ with the usual round metric and which have instanton number $N=1$. The construction takes the instanton on $\mathbb{R}^{4}$ with unit scale size and makes a stereographic projection to $S^{4}$. The gauge field of an $S U(2)$ instanton located at the origin in $\mathbb{R}^{4}$, with scale size $\mu$, is given by 52

$$
i A_{\mu}(x)=\frac{|x|}{\mu^{2}+|x|^{2}}\left(x^{4}+i x^{a} \sigma_{a}\right) \partial_{\mu}\left(\frac{x^{4}-i x^{a} \sigma_{a}}{|x|}\right),
$$

where $\sigma_{a}(a=1,2,3)$ denotes the Pauli matrices, interpreted as $S U(2)$ generators acting on the fundamental representation. The explicit components then read

$$
A_{a}(x)=\frac{\epsilon_{a b c} x^{c} \sigma_{b}-x^{4} \sigma_{a}}{\mu^{2}+|x|^{2}}, \quad A_{4}(x)=\frac{x^{a} \sigma_{a}}{\mu^{2}+|x|^{2}},
$$

while the corresponding field strength is

$$
F_{a b}=\frac{2 \mu^{2} \epsilon_{a b c} \sigma_{c}}{\left(\mu^{2}+|x|^{2}\right)^{2}}, \quad F_{a 4}=\frac{2 \mu^{2} \sigma_{a}}{\left(\mu^{2}+|x|^{2}\right)^{2}}
$$

which is explicitly self-dual.

The stereographic projection from $\mathbb{R}^{4}$ to $S^{4}$ is accomplished by the map

$$
\begin{aligned}
x^{1} & =\cot \left(\alpha^{1} / 2\right) c_{2}, \\
x^{2} & =\cot \left(\alpha^{1} / 2\right) s_{2} c_{3}, \\
x^{3} & =\cot \left(\alpha^{1} / 2\right) s_{2} s_{3} c_{4}, \\
x^{4} & =\cot \left(\alpha^{1} / 2\right) s_{2} s_{3} s_{4},
\end{aligned}
$$

where $s_{i} \equiv \sin \left(\alpha^{i}\right), c_{i} \equiv \cos \left(\alpha^{i}\right)$ and the angles on the four-sphere are defined in eq. (38). Performing this coordinate transformation for the $\mu=1$ instanton, we find the explicit field strengths

$$
\begin{aligned}
\tilde{F}_{12} & =\frac{\lambda}{2} s_{1}\left[s_{3} s_{4} \sigma_{1}-s_{3} c_{4} \sigma_{2}+c_{3} \sigma_{3}\right] \\
\tilde{F}_{13} & =\frac{\lambda}{2} s_{1} s_{2}\left[\left(s_{2} c_{4}+c_{2} c_{3} s_{4}\right) \sigma_{1}+\left(s_{2} s_{4}-c_{2} c_{3} c_{4}\right) \sigma_{2}-c_{2} s_{3} \sigma_{3}\right] \\
\tilde{F}_{14} & =\frac{\lambda}{2} s_{1} s_{2} s_{3}\left[\left(c_{2} c_{4}-s_{2} c_{3} s_{4}\right) \sigma_{1}+\left(c_{2} s_{4}+s_{2} c_{3} c_{4}\right) \sigma_{2}+s_{2} s_{3} \sigma_{3}\right]
\end{aligned}
$$

where as in Section 4 , we use the definition $\tilde{F}_{i j} \equiv \lambda F_{\alpha^{i} \alpha^{j}}$. The remaining components of the field strength follow from self-duality, e.g., $\tilde{F}_{34}=\sqrt{g} g^{11} g^{22} \tilde{F}_{12}=s_{1} s_{2}^{2} s_{3} \tilde{F}_{12}$. This configuration manifestly satisfies the condition for a homogeneous instanton (45) with $N=1$,

$$
\operatorname{Tr} \tilde{F}_{i j}^{*} \tilde{F}^{i j}=6 \lambda^{2}
$$

\footnotetext{
${ }^{3}$ We would like to acknowledge Jan Segert for invaluable assistance on the material presented in the following.
} 
In fact, we note that as required at various steps in the analysis of the D5-brane spike, this expression is proportional to the gauge identity matrix, i.e., $\tilde{F}_{i j}{ }^{*} \tilde{F}^{i j}=3 \lambda^{2} \mathbf{1}_{2}$.

The above gauge fields can be extended to a homogeneous instanton for the gauge group $S U(n)$ by simply replacing the $\sigma_{a}$ in eqs. (90) and (92) by an $n \times n$ matrix representation of the $S U(2)$ algebra (12). The latter can then be interpreted as $S U(n)$ generators acting in the fundamental representation. If we choose the irreducible $n \times n$ representation, the corresponding gauge field satisfies

$$
\tilde{F}_{i j}^{*} \tilde{F}^{i j}=\left(n^{2}-1\right) \lambda^{2} \mathbf{1}_{n}
$$

corresponding to instanton number (and D-string number)

$$
N=\frac{n\left(n^{2}-1\right)}{6}
$$

A further result is that the homogeneous $S U(n)$ gauge field with the maximum possible instanton number is in fact the above configuration constructed with the irreducible $n \times n$ representation of $S U(2)$. The proof, which we sketch below, relies on two mathematical results. The first is is the classification of homogeneous principal bundles, and the second is the classification of symmetric connections on the homogeneous principal bundles, by a theorem of H.C. Wang [32].

In general, we wish to consider principal fibre bundles which admit a fibre-transitive Lie group of automorphisms, which will be a necessary ingredient for Wang's theorem. Let $P$ be a principal $H$ bundle and $M=P / H$ is the base space. Let $G$ be the automorphism group which acts on $P$ by left multiplication. These transformations commute with the $H$ action (on the right), and so induce a $G$ action on $M$. Suppose that this $G$ action on $M$ is transitive, and that $G_{0}$ is the stabilizer at some point $x$ in $M$. Then $G_{0}$ acts on the fibre over $x$, commuting with the $H$ action. Since the fibre of the principal bundle is isomorphic to the group $H$, the $G_{0}$ action must be via a homomorphism $\phi: G_{0} \rightarrow H$. Thus the homogeneous principal bundles are in one-to-one correspondence with the latter homomorphisms. For the particular case at hand, we consider $H=S U(2), M=S^{4}$ and $G=\operatorname{Spin}(5)=S p(4)$. Now the stabilizer is $G_{0}=\operatorname{Spin}(4) \simeq$ $S U(2) \times S U(2)$, and hence we consider homomorphisms $\phi: S U(2) \times S U(2) \rightarrow S U(2)$. Aside from the trivial case, there are two homomorphisms: one which is the identity map on the first factor and trivial on the second, and another which switches the role of the two $S U(2)$ factors. Only one of these supports a self-dual connection (the other is anti-self-dual), and it corresponds to the quaternionic Hopf fibration of the seven-sphere [53], i.e., $S^{7}$ as $S U(2)=S^{3}$ fibred over $S^{4}$. For a principal $S U(n)$ bundle, we have the homomorphisms $\phi: S U(2) \times S U(2) \rightarrow S U(n)$. Now these homomorphisms will split as $\phi_{1}: S U(2) \rightarrow K_{1} \subset S U(n)$ and $\phi_{2}: S U(2) \rightarrow K_{2} \subset S U(n)$. Hence all of the homogeneous (Spin(5) invariant) bundles will decompose as bundles associated with the Hopf and anti-Hopf fibrations. Now given a $G$-invariant principal $H$ bundle, Wang's theorem [32] puts the invariant connections over these bundles in one-to-one correspondence with certain linear mappings from the Lie algebras of $G \rightarrow H$. Essentially these are the mappings consistent with the homomorphisms identified above. Hence in the case of interest, identifying the invariant connections corresponds to identifying $S U(2)$ subgroups of $S U(n)$. That is, the above construction in fact includes all of the homogeneous gauge configurations 
on the four-sphere. It is straightforward to show then that the instanton number is maximized by the choice of the irreducible $n \times n$ representation.

\section{References}

[1] J. Polchinski, Phys. Rev. Lett. 75, 4724 (1995), hep-th/9510017;

J. Dai, R. G. Leigh and J. Polchinski, Mod. Phys. Lett. A4, 2073 (1989).

[2] J. Polchinski, "TASI lectures on D-branes," hep-th/9611050.

[3] C. V. Johnson, "D-brane primer," hep-th/0007170.

[4] R. G. Leigh, Mod. Phys. Lett. A4, 2767 (1989).

[5] A.A. Tseytlin, "Born-Infeld action, supersymmetry and string theory," in The many faces of the superworld, ed. M. Shifman (World Scientific, 2000), hep-th/9908105.

[6] C.G. Callan and J.M. Maldacena, Nucl. Phys. B513, 198 (1998), hep-th/9708147;

G.W. Gibbons, Nucl. Phys. B514, 603 (1998), hep-th/9709027;

P.S. Howe, N.D. Lambert and P.C. West, Nucl. Phys. B515, 203 (1998), hep-th/9709014.

[7] T. Banks, W. Fischler, S.H. Shenker and L. Susskind, Phys. Rev. D55, 5112 (1997), hepth/9610043.

[8] D. Kabat and W. Taylor, Adv. Theor. Math. Phys. 2, 181 (1998), hep-th/9711078;

S. Rey, "Gravitating M(atrix) Q balls," hep-th/9711081.

[9] R.C. Myers, JHEP 9912, 022 (1999), hep-th/9910053.

[10] E. Witten, Nucl. Phys. B460, 335 (1996), hep-th/9510135.

[11] W. Taylor and M. Van Raamsdonk, Nucl. Phys. B573 (2000) 703, hep-th/9910052.

[12] J. McGreevy, L. Susskind and N. Toumbas, JHEP 0006, 008 (2000), hep-th/0003075.

[13] J. Polchinski and M.J. Strassler, "The string dual of a confining four-dimensional gauge theory," hep-th/0003136.

[14] N.R. Constable, R.C. Myers and Ø. Tafjord, Phys. Rev. D61, 106009 (2000), hepth/9911136.

[15] N. D. Lambert and I. Sachs, JHEP 0008, 024 (2000), hep-th/0006122.

[16] N.D. Lambert and D. Tong, Phys. Lett. B462, 89 (1999), hep-th/9907014;

M. Zamaklar, "Geometry of the DBI dyonic instanton," hep-th/0006090.

[17] S. P. Trivedi and S. Vaidya, JHEP 0009, 041 (2000), hep-th/0007011. 
[18] C. Bachas, J. Hoppe and B. Pioline, "Nahm equations, $N=1^{*}$ domain walls, and D-strings in $A d S_{5} \times S^{5}$," hep-th/0007067.

[19] J. Kluson, JHEP 0011, 016 (2000), hep-th/0009189.

[20] Y. Lozano, "Non-commutative branes from M-theory," hep-th/0012137.

[21] A. Strominger, Phys. Lett. B383, 44 (1996), hep-th/9512059;

P.K. Townsend, Nucl. Phys. Proc. Suppl. 58, 163 (1997), hep-th/9609217.

[22] G.W. Semenoff, K. Zarembo, Nucl. Phys. 556, 247 (1999), hep-th/9903140.

[23] D.-E. Diaconescu, Nucl. Phys. B503, 220 (1997), hep-th/9608163.

[24] J. Castelino, S. Lee and W. Taylor, Nucl. Phys. B526, 334 (1998), hep-th/9712105.

[25] J.P. Gauntlett, J. Gomis, P.K. Townsend, JHEP 9801, 003 (1998), hep-th/9711205;

D. Brecher, Phys. Lett. B442, 117 (1998), hep-th/9804180.

[26] S. Lee, A. Peet and L. Thorlacius, Nucl. Phys. B514, 161 (1998), hep-th/9710097;

D. Kastor and J. Traschen, Phys. Rev. D61, 024034 (2000), hep-th/9906237.

[27] A.A. Tseytlin, Nucl. Phys. B501, 41 (1997), hep-th/9701125.

[28] W. Nahm, Phys. Lett. B90, 413 (1980);

W. Nahm, "The construction of all self-dual multimonopoles by the ADHM method," in "Monopoles in quantum field theory," Craigie et al. (eds), World Scientific, Singapore (1982).

[29] J. Hoppe, Quantum Theory of a Massless Relativistic Surface and a Two-Dimensional Bound State Problem, MIT Ph.D. thesis, 1982 (available at http://www.aeipotsdam.mpg.de/ hoppe); Elem. Part. Res. J. (Kyoto) 80, 145 (1989);

B. de Wit, J. Hoppe and H. Nicolai, Nucl. Phys. B305, 545 (1988);

J. Madore, An Introduction of Noncommutative Differential Geometry and its Applications, Cambridge University Press, Cambridge, 1995.

[30] H. Grosse, C. Klimcik and P. Presnajder, Commun. Math. Phys. 180, 429 (1996), hepth/9602115.

[31] M.F. Atiyah, "Geometry Of Yang-Mills Fields," in "Collected works, vol. 5*", pp 75-173, Oxford University Press (1988).

[32] H.C. Wang, Nagoya. Math. J. 13, 1 (1958).

[33] J.H. Schwarz, Phys. Lett. B360, 13 (1995), hep-th/9508143.

[34] M. Li, Nucl. Phys. B460, 351 (1996), hep-th/9510161.

[35] A.W. Peet, unpublished. 
[36] J. Maldacena, Adv. Theor. Math. Phys. 2, 231 (1998), hep-th/9711200;

For a review, see: O. Aharony, S.S. Gubser, J. Maldacena, H. Ooguri and Y. Oz, Phys. Rept. 323, 183 (2000), hep-th/9905111.

[37] A. Connes, Noncommutative Geometry, Academic Press (1994).

[38] L. Freidel and K. Krasnov, unpublished.

[39] E. Hawkins, "Noncommutative regularization for the practical man," hep-th/9908052.

[40] A. Hashimoto and W. Taylor, Nucl. Phys. B503, 193 (1997), hep-th/9703217;

F. Denef, A. Sevrin and J. Troost, Nucl. Phys. B581, 135 (2000), hep-th/0002180.

[41] P. Bain, "On the non-Abelian Born-Infeld action," hep-th/9909154; A. Sevrin, J. Troost and W. Troost, "The non-abelian Born-Infeld action at order $F^{6}$," hep-th/0101192.

[42] C.P. Bachas and P. Bain, unpublished; A. Hashimoto and R.C. Myers, unpublished.

[43] For a review, see: W. Taylor, "M(atrix) theory: Matrix quantum mechanics as a fundamental theory," hep-th/0101126.

[44] R. Dijkgraaf, E. Verlinde and H. Verlinde, Nucl. Phys. B500, 43 (1997), hep-th/9703030.

[45] R. Schiappa, "Matrix strings in weakly curved background fields," hep-th/0005145.

[46] A. Hashimoto, Phys. Rev. D57, 6441 (1998), hep-th/9711097.

[47] N. J. Hitchin, Commun. Math. Phys. 83, 579 (1982);

See also: N.S. Manton and M.K. Murray, "Symmetric Monopoles", hep-th/9407102.

[48] V. Balasubramanian and R.G. Leigh, Phys. Rev. D55, 6415 (1997), hep-th/9611165.

[49] J.C. Breckenridge, G. Michaud and R.C. Myers, Phys. Rev. D55, 6438 (1997), hepth/9611174;

M.S. Costa and G. Papadopoulos, Nucl. Phys. B510, 217 (1998), hep-th/9612204.

[50] N. Itzhaki, J.M. Maldacena, J. Sonnenschein and S. Yankielowicz, Phys. Rev. D58, 046004 (1998), hep-th/9802042.

[51] E. Corrigan, C. Devchand, D.B. Fairlie and J. Nuyts, Nucl. Phys. B214, 452 (1983);

L. Baker and D. Fairlie, J. Math. Phys. 40, 2539 (1999), hep-th/9901072.

[52] A.A. Belavin, A.M. Polyakov, A.S. Schwarz and Y.S. Tyupkin, Phys. Lett. B59, 85 (1975).

[53] See, for example: M. Nakahara, "Geometry, Topology and Physics" (Adam Hilger, 1990). 